\title{
A DECOMPOSITION TECHNIQUE FOR JOHN DOMAINS
}

\section{Lars Diening, Michael Růžička and Katrin Schumacher}

\author{
Freiburg University, Section of Applied Mathematics \\ Eckerstrasse 1, D-79104 Freiburg, Germany; diening@mathematik.uni-freiburg.de \\ Freiburg University, Section of Applied Mathematics \\ Eckerstrasse 1, D-79104 Freiburg, Germany; rose@mathematik.uni-freiburg.de \\ Technische Universität Darmstadt, Department of Mathematics \\ Schlossgartenstrasse 7, D-64289 Darmstadt, Germany; schumacher@mathematik.tu-darmstadt.de
}

\begin{abstract}
We develop a method to decompose functions with mean value zero that are defined on a (possibly unbounded) John domain into a countable sum of functions with mean value zero and support in cubes or balls. This method enables us to generalize results known for simple domains to the class of John domains and domains satisfying a certain chain condition. As applications we present the solvability of the divergence equation $\operatorname{div} \mathbf{u}=f$, the negative norm theorem, Korn's inequality, Poincaré's inequality and a localized version of the Fefferman-Stein inequality. We present the results for weighted Lebesgue spaces and Orlicz spaces.
\end{abstract}

\section{Introduction}

In this paper we present a technique that allows to generalize results known for simple domains such as balls or cubes to very general domains. The natural class of domains to which this method is applicable is the one of John domains. The concept of (bounded) John domains has been introduced by John in [21] and named after him by Martio and Sarvas in [23], who also introduced the concept of unbounded John domains. We shall use the terminology of Näkki and Väisälä [24], who gave an exposition of the basic theory of John domains. Roughly speaking, a domain is a John domain if it is possible to travel from one point of the domain to another without getting too close to the boundary. This class contains Lipschitz domains but is much larger. John domains may possess fractal boundaries or internal cusps while external cusps are excluded. Bounded John domains can also be characterized by the Boman chain condition. We generalize this idea to the case of unbounded John domains and present our result assuming the more general emanating chain condition. In particular, aperture domains and domains with conical outlets satisfy this condition. The precise definitions of John domains, the Boman chain condition, and the emanating chain condition are given in section 3 .

The relation between these three conditions is the following (see Remarks 3.10, 3.16, and 3.17):

bounded domain: John = Boman chain = emanating chain

unbounded domain: John $=$ Boman chain $\varsubsetneqq$ emanating chain

doi:10.5186/aasfm.2010.3506

2000 Mathematics Subject Classification: Primary 26D10, 46E30, 35Q35.

Key words: John domains, decomposition, divergence equation, negative norm theorem, Korn's inequality, Poincaré's inequality, Fefferman-Stein inequality, weighted Lebesgue spaces, Orlicz spaces, Boman chain condition. 
The idea of our method is the following. Let $\Omega$ be a domain satisfying the emanating chain condition and $1<q<\infty$. We consider a function $f \in L_{0}^{q}(\Omega)$, i.e. $f$ is $q$ integrable and has vanishing mean if $\Omega$ is bounded. We decompose the domain $\Omega$ by the Whitney method into cubes (or balls) $W_{i}$. Then we decompose $f$ in $L_{0}^{q}(\Omega)$ as the sum of functions $g_{i} \in L_{0}^{q}\left(W_{i}\right)$ such that

$$
\left(\sum_{i \in \mathbf{N}}\left\|g_{i}\right\|_{L_{0}^{q}\left(W_{i}\right)}^{q}\right)^{\frac{1}{q}} \leq c\|f\|_{L_{0}^{q}(\Omega)} .
$$

The functions $g_{i}$ are extended outside of $W_{i}$ by zero.

We show that the decomposition technique described above is also applicable in the weighted setting, where $L_{0}^{q}(\Omega)$ and $L_{0}^{q}\left(W_{i}\right)$ are replaced by their weighted counterparts $L_{w, 0}^{q}(\Omega)$ and $L_{w, 0}^{q}\left(W_{i}\right)$, respectively. The biggest class of weight functions to which our proofs apply is the one of Muckenhoupt weights, see Definition 2.2 below. The reason is that the continuity of the decomposition operator is based on the continuity of the Hardy-Littlewood maximal operator $M$ on $L_{w}^{q}(\Omega)$, which requires that $w$ is in the Muckenhoupt class $A_{q}$, see Theorem 2.4. The presence of the weight function barely complicates the proofs in this article. However, it increases the significance of our results. The reason is that the results can be easily generalized by the powerful extrapolation technique of Rubio de Francia [26] from weighted Lebesgue spaces to a large class of Banach function spaces. As an example we apply this technique to generalize our results to the setting of Orlicz spaces. We need these results in a forthcoming article for the numerical analysis of the $q$-Stokes system.

We propose several important applications of our decomposition method. First we give a proof of Poincaré's inequality. Then we consider the existence of a solution $\mathbf{u} \in\left(D_{w, 0}^{1, q}(\Omega)\right)^{n}$ of the divergence equation $\operatorname{div} \mathbf{u}=f$ on $\Omega$ satisfying $\|\nabla \mathbf{u}\|_{L_{w}^{q}(\Omega)} \leq$ $c\|f\|_{L_{w, 0}^{q}(\Omega)}$ for a given function $f \in L_{w, 0}^{q}(\Omega)$. This is a famous auxiliary problem and fundamental tool in fluid dynamics. The solvability of the divergence equation is now a straightforward consequence of the corresponding result for Lipschitz domains $[15,27]$ and of the above decomposition. As a consequence we are able the generalize Nečas theorem on negative norms (Lions-Lemma) and Korn's inequality to the case of domains satisfying the emanating chain condition.

Next, we apply the decomposition technique to obtain a weighted, localized version of the famous Fefferman-Stein inequality [13] for bounded domains satisfying the emanating chain condition. In particular, we show that

$$
\left\|f-\langle f\rangle_{\Omega}\right\|_{L_{w}^{q}(\Omega)} \leq c\left\|M_{\mathrm{res}, \Omega, \sigma_{1}}^{\sharp} f\right\|_{L_{w}^{q}(\Omega)} \text { for every } f \in L^{1}(\Omega),
$$

where $M_{\mathrm{res}, \Omega, \sigma_{1}}^{\sharp}$ is the restricted sharp maximal operator and $\sigma_{1} \in[1, \infty)$, see Section 5.3.

\section{Preliminaries}

Let us start with the necessary notation and convention we use in the paper. By $c$ we denote a generic constant, i.e. its value may change from line to line, but does not depend on the important quantities. We write $f \sim g$ if there exist constants $c_{1}, c_{2}>0$ so that $c_{1} f \leq g \leq c_{2} g$. We use $\delta_{j, k}$ for the Kronecker delta, i.e. $\delta_{j, k}=0$ for $j \neq k$ and 
$\delta_{j, j}=1$. The set of non-negative real numbers is denoted by $\mathbf{R}^{\geq}$. For an open (nonempty) set $\Omega \subset \mathbf{R}^{n}$ we denote by $L^{0}(\Omega)$ the space of real-valued, Lebesgue measurable functions on $\Omega$ and by $L_{\text {loc }}^{1}(\Omega)$ the space of real-valued, locally integrable functions on $\Omega$. We understand "locally integrable" in the sense that $f$ is integrable on every compact subset of $\Omega$. We equip $L_{\text {loc }}^{1}(\Omega)$ with the usual topology of $L^{1}$-convergence on compact subsets. By $C_{0}^{\infty}(\Omega)$ we denote the space of smooth functions with compact support. The set $C_{0,0}^{\infty}(\Omega)$ is the set of functions $f \in C_{0}^{\infty}(\Omega)$ with $\int_{\Omega} f(x) d x=0$. An open connected set $\Omega \subset \mathbf{R}^{n}$ is called a domain. We use $\langle f, g\rangle$ for the duality pairing and for $\int_{\mathbf{R}^{n}} f(y) g(y) d y$. By $l^{q}:=l^{q}\left(\mathbf{N}_{0}\right)$ we denote the sequence Lebesgue space with $q$-summability. We use $\chi_{E}$ for the characteristic function of a Lebesgue measurable set $E \subset \mathbf{R}^{n}$. By $L^{q}(\Omega)$ and $W^{1, q}(\Omega)$ with $q \in[1, \infty]$ we denote the usual Lebesgue and Sobolev spaces, respectively. We use $|\Omega|$ for the $n$-dimensional Lebesgue measure of $\Omega$ and $\operatorname{diam}(\Omega)$ as the diameter of $\Omega$. By $B_{r}(x)$ we denote the ball in $\mathbf{R}^{n}$ with center $x$ and radius $r$. For $f \in L^{1}(E)$ with $|E|>0$ we denote the mean value of $f$ over $E$ by

$$
\langle f\rangle_{E}:=f_{E} f(y) d y:=\frac{1}{|E|} \int_{E} f(y) d y .
$$

Throughout the paper all cubes have sides parallel to the axes. Moreover, all cubes and balls are open sets. If $f \in L^{1}(E)$ for some Lebesgue measurable set $E \subset \mathbf{R}^{n}$, then we often implicitly extend $f$ outside of $E$ by zero, so that $f \in L^{1}\left(\mathbf{R}^{n}\right)$. For a cube $Q \subset \mathbf{R}^{n}$ and $\lambda>0$ we denote by $\lambda Q$ the cube with the same center as $Q$ and $\lambda$-times the diameter of $Q$.

For $f \in L_{\text {loc }}^{1}\left(\mathbf{R}^{n}\right)$ we define the maximal operator $M$ by

$$
(M f)(x)=\sup _{Q \ni x} f_{Q}|f(y)| d y
$$

where the supremum is taken over all cubes $Q$ in $\mathbf{R}^{n}$ that contain $x$.

Definition 2.2. For $1<q<\infty$ let $A_{q}$, the set of Muckenhoupt weights, be given by all $w \in L_{\text {loc }}^{1}\left(\mathbf{R}^{n}\right)$ with $w>0$ almost everywhere and

$$
A_{q}(w):=\sup _{Q}\left[\left(f_{Q} w d x\right)\left(f_{Q} w^{-\frac{1}{q-1}} d x\right)^{q-1}\right]<\infty .
$$

The supremum is taken over all cubes in $\mathbf{R}^{n}$. The value $A_{q}(w)$ is called the $A_{q^{-}}$ constant of $w$.

The class $A_{\infty}$ is defined by $A_{\infty}=\bigcup_{q>1} A_{q}$. For $w \in A_{\infty}$ we define the $A_{\infty}$ constant $A_{\infty}(w)$ by the limit of $A_{q}(w)$ as $q \rightarrow \infty$. This limit is well defined, since $A_{q}(w)$ is non-increasing for $q \rightarrow \infty$.

The class $A_{1}$ is the set of all $w \in L_{\text {loc }}^{1}\left(\mathbf{R}^{n}\right)$ with $w>0$ almost everywhere and

$$
A_{1}(w):=\sup _{Q}\left[f_{Q} w d x \sup _{x \in Q} \frac{1}{w(x)}\right]<\infty .
$$

For convenience of notation we write $w(E):=\int_{E} w d x$ for every $w \in A_{q}$ with $1 \leq q \leq \infty$ and every Lebesgue measurable set $E \subset \mathbf{R}^{n}$. 
A constant $C=C(w)$ is called $A_{q}$-consistent if it depends on $w$ only via $A_{q}(w)$. For $w \in A_{q}$ and an open set $\Omega \subset \mathbf{R}^{n}$ we define

$$
L_{w}^{q}(\Omega):=\left\{f \in L_{\mathrm{loc}}^{1}(\Omega):\|f\|_{L_{w}^{q}(\Omega)}:=\left(\int_{\Omega}|f|^{q} w d x\right)^{\frac{1}{q}}<\infty\right\} .
$$

It is easy to verify that $\left(L_{w}^{q}(\Omega)\right)^{\prime}=L_{w^{\prime}}^{q^{\prime}}(\Omega)$ with $\frac{1}{q}+\frac{1}{q^{\prime}}=1$ and $w^{\prime}:=w^{-\frac{1}{q-1}} \in A_{q^{\prime}}$ for $1<q<\infty$. Moreover, $L_{w}^{q}(\Omega) \hookrightarrow L^{1}(\Omega)$ for every bounded $\Omega$ using Hölder's inequality and (2.3).

One has the following well-known close connection between the Muckenhoupt class $A_{q}$ and the maximal operator.

Theorem 2.4. Let $1<q<\infty$ and $w \in A_{q}$. Then the maximal operator $M$ is continuous on $L_{w}^{q}\left(\mathbf{R}^{n}\right)$. More precisely, there exists an $A_{q}$-consistent constant $c$ such that

$$
\|M f\|_{L_{w}^{q}\left(\mathbf{R}^{n}\right)} \leq c\|f\|_{L_{w}^{q}\left(\mathbf{R}^{n}\right)} \quad \text { for every } f \in L_{w}^{q}\left(\mathbf{R}^{n}\right) .
$$

Proof. See [14], Theorems 2.1 and 2.9. For the $A_{q}$-consistence of the constants one has to re-read the proof of [14], Theorem 2.9.

Let $\Omega \subset \mathbf{R}^{n}$ be a domain, $1 \leq q<\infty$, and $w \in A_{q}$. We define

$$
W_{w}^{1, q}(\Omega):=\left\{u \in L_{w}^{q}(\Omega):\|u\|_{W_{w}^{1, q}(\Omega)}:=\|u\|_{L_{w}^{q}(\Omega)}+\|\nabla u\|_{L_{w}^{q}(\Omega)}<\infty\right\},
$$

where $\nabla u$ is the weak derivative of $u$. Moreover, we set

$$
W_{w, 0}^{1, q}(\Omega)={\overline{C_{0}^{\infty}(\Omega)}}^{\|\cdot\|_{W_{w}^{1, q}(\Omega)}} .
$$

\section{John domains and the emanating chain condition}

3.1. John domains. We use John domains in the sense of Martio, Sarvas [23] and Näkki, Väisälä [24]. This includes the case of unbounded John domains. Several equivalent characterizations for John domains can be found in [24]. In the present paper we shall adopt the definition based on cigars and carrots.

Let $\gamma \subset \mathbf{R}^{n}$ be a rectifiable path with endpoints $a$ and $b$ and length $|\gamma|$. We assume that all our paths are parameterized by its arclength. In particular, $\gamma:[0,|\gamma|] \rightarrow$ $\mathbf{R}^{n}$. We define the $\alpha$-cigar with core $\gamma$ and parameter $\alpha>0$ by

$$
\operatorname{cig}(\gamma, \alpha):=\bigcup_{t \in[0,|\gamma|]}\left\{B\left(\gamma(t), \frac{1}{\alpha} \min \{t,|\gamma|-t\}\right)\right\} .
$$

Further, we define the $\beta$-carrot with core $\gamma$ and parameter $\beta>0$ by

$$
\operatorname{car}(\gamma, \beta):=\bigcup_{t \in[0,|\gamma|]}\left\{B\left(\gamma(t), \frac{1}{\beta} t\right)\right\} .
$$

Definition 3.1. A domain $\Omega \subset \mathbf{R}^{n}$ is called an $\alpha$-John domain, $\alpha>0$, if every pair of distinct points $a, b \in \Omega$ can be joined by a rectifiable path $\gamma$ such that $\operatorname{cig}(\gamma, \alpha) \subset \Omega$.

If the constant $\alpha$ is not important, we just say that $\Omega$ is a John domain.

John domains may possess fractal boundaries or internal cusps while external cusps are excluded. For example the interior of Koch's snow flake is a John domain. The half space $\left\{\left(x_{1}, \ldots, x_{n}\right) \in \mathbf{R}^{n}: x_{n}>0\right\}$ and the whole space $\mathbf{R}^{n}$ are also John 
domains, while the aperture domain $\left\{\left(x_{1}, x_{2}\right) \in \mathbf{R}^{n}: x_{2} \neq 0\right.$ or $\left.\left|x_{1}\right|<1\right\}$ is not a John domain. We will see later that our technique still applies to this kind of domains, since it satisfies (see Remark 3.17) the emanating chain condition, which is defined below.

Remark 3.2. If $\Omega$ is bounded, then Definition 3.1 is equivalent to several other definitions (cf. [24]). In particular, if $\Omega$ is a bounded, $\alpha$-John domain, then there exists $x_{0} \in \Omega$ (the John center) and $\beta=\beta(\alpha)>0$ such that each $a \in \Omega \backslash\left\{x_{0}\right\}$ can be joined to $x_{0}$ by a rectifiable path $\gamma$ such that $\operatorname{car}(\gamma, \beta) \subset \Omega$.

3.2. The emanating chain condition. We show now that $\alpha$-John domains can be decomposed into a suitable set of balls or cubes that satisfy a certain chain condition.

It can be shown (cf. [30, Chapter 6], [9, Chapter 1], [6, Theorem 3.2], [28, Section 2], [11, Lemma C.1]) that every proper, open subset $\Omega \subset \mathbf{R}^{n}$ possesses a suitable Whitney decomposition, which we summarize in the following proposition.

Proposition 3.3. There exist $\kappa_{1}, \kappa_{2}$ with $1<\kappa_{1}<\kappa_{2}$ and $N>0$ such that for every open set $\Omega \subset \mathbf{R}^{n}$ with $\Omega \neq \mathbf{R}^{n}$ there exists a family $Q_{j}, j \in \mathbf{N}_{0}$, of cubes (or balls) such that

(W1) $\Omega=\bigcup_{j \in \mathbf{N}_{0}} \kappa_{1} Q_{j}=\bigcup_{j \in \mathbf{N}_{0}} 2 \kappa_{1} Q_{j}$,

(W2) $\frac{1}{2} \kappa_{1} \operatorname{diam}\left(Q_{j}\right) \leq \operatorname{dist}\left(Q_{j}, \partial \Omega\right) \leq \kappa_{2} \operatorname{diam}\left(Q_{j}\right)$,

(W3) $\sum_{j \in \mathbf{N}_{0}} \chi_{2 \kappa_{1} Q_{j}} \leq N \chi_{\Omega}$ on $\mathbf{R}^{n}$.

A family of cubes (or balls) $\left\{Q_{j}\right\}$ which satisfies (W1)-(W3) is called a Whitney covering of $\Omega$ with constants $\kappa_{1}, \kappa_{2}, N$. Two different cubes from a Whitney covering are called neighbors if their closures intersect each other.

Remark 3.4. Due to (W3) every cube from a Whitney covering has at most $c(N)$ neighbors. Moreover, if follows from (W2) that two neighbors have comparable size. Indeed, if $Q_{1}$ and $Q_{2}$ are neighbors and $y \in Q_{1} \cap Q_{2}$, then it follows from (W2) that $\operatorname{diam}\left(Q_{1}\right) \sim \operatorname{dist}(y, \partial \Omega) \sim \operatorname{diam}\left(Q_{2}\right)$ with constants depending on $\kappa_{1}, \kappa_{2}, N$, and $n$.

Let us first treat the case of a bounded John domain. For this we introduce a new chain condition.

Definition 3.5. Let $\Omega \subset \mathbf{R}^{n}$ be a bounded domain and let $\sigma_{1}, \sigma_{2} \geq 1$. Then we say that $\Omega$ satisfies the emanating chain condition with constants $\sigma_{1}$ and $\sigma_{2}$ if there exists a covering $\mathscr{W}=\left\{W_{i}: i \in \mathbf{N}_{0}\right\}$ of $\Omega$ consisting of open cubes (or balls) such that:

(C1) We have $\sigma_{1} W \subset \Omega$ for all $W \in \mathscr{W}$ and $\sum_{W \in \mathscr{W}} \chi_{\sigma_{1} W} \leq \sigma_{2} \chi_{\Omega}$ on $\mathbf{R}^{n}$.

(C2) For every $W_{i} \in \mathscr{W}$ there exists a chain of (pairwise different) $W_{i, 0}, W_{i, 1}, \ldots$, $W_{i, m_{i}}$ from $\mathscr{W}$ such that $W_{i, 0}=W_{i}, W_{i, m_{i}}=W_{0}$, and $W_{i, k_{1}} \subset \sigma_{2} W_{i, k_{2}}$ for $0 \leq k_{1} \leq k_{2} \leq m_{i}$. Moreover, $W_{i, k} \cap W_{i, k+1}, 0 \leq k<m_{i}$, contains a ball $B_{i, k}$ such that $W_{i, k} \cup W_{i, k+1} \subset \sigma_{2} B_{i, k}$. The chain $W_{i, 0}, \ldots, W_{i, m_{i}}$ is called chain emanating from $W_{i}$. The number $m_{i} \in \mathbf{N}_{0}$ is called the length of this chain.

(C3) The set $\left\{i \in \mathbf{N}_{0}: W_{i} \cap K \neq \emptyset\right\}$ is finite for every compact subset $K \subset \Omega$.

The family $\mathscr{W}$ is called the chain-covering of $\Omega$. The cube $W_{0}$ is called the central cube (or ball), since every chain ends in $W_{0}$. 
Remark 3.6. Let $\Omega$ be a bounded domain satisfying the emanating chain condition with constants $\sigma_{1}$ and $\sigma_{2}$ and central cube $W_{0}$. Then $\Omega \subset \sigma_{2} W_{0}$ and hence $\operatorname{diam}\left(W_{0}\right) \leq \operatorname{diam}(\Omega) \leq \sigma_{2} \operatorname{diam}\left(W_{0}\right)$. This follows from the fact that every chain ends in $W_{0}$ and is therefore contained in $\sigma_{2} W_{0}$.

Remark 3.7. Domains $\Omega$ satisfying only the requirements $(\mathrm{C} 1)$ and $(\mathrm{C} 2)$ in Definition 3.5 are known as domains satisfying the Boman chain condition. The concept of a Boman chain condition appears to our knowledge first in the article of Iwaniec and Nolder [20], where they refer to a preprint of Boman [3]. A related decomposition can be found in a paper by Hurri [16].

It has been shown by Buckley, Koskela, and Lu [4] that a bounded domain is a John domain if and only if it satisfies the Boman chain condition. We now improve this statement slightly in proving that a bounded John domain satisfies the emanating chain condition. We also need this proof later for the case of unbounded domains.

Theorem 3.8. Let $\Omega \subset \mathbf{R}^{n}$ be a bounded $\alpha$-John domain with center $x_{0}$ and let $\left\{Q_{i}\right\}_{i \in \mathbf{N}_{0}}$ be a Whitney covering of $\Omega$ with constants $\kappa_{1}, \kappa_{2}, N$ such that $x_{0} \in Q_{0}$. Let $\sigma_{1}:=\frac{4}{3}$. Define $W_{i}:=\frac{3}{2} \kappa_{1} Q_{i}$ for $i \in \mathbf{N}_{0}$ and $\mathscr{W}:=\left\{W_{i}: i \in \mathbf{N}_{0}\right\}$. Then there exists $\sigma_{2}=\sigma_{2}\left(\kappa_{1}, \kappa_{2}, N, \alpha, n\right) \geq 1$ such that $\Omega$ satisfies the emanating chain condition with constants $\sigma_{1}$ and $\sigma_{2}$ and chain-covering $\mathscr{W}$.

Proof. We use the characterization of Remark 3.2 of bounded $\alpha$-John domains. So let $\beta>0$ be as in Remark 3.2. For every $j \in \mathbf{N}$ let $x_{j}$ denote the center of $W_{j}$ defined in the theorem. We do not need that $x_{0}$ is the center of $W_{0}$. However, it is possible to show that the center of $W_{0}$ is also a John center of $\Omega$, so we could replace $x_{0}$ by the center of $W_{0}$. This could possibly change the value of $\alpha$ by a fixed constant depending on $\kappa_{1}$. We set $\mathscr{W}:=\left\{W_{i}: i \in \mathbf{N}_{0}\right\}$.

For every $i \in \mathbf{N}_{0}$ we construct a finite sequence $Q_{i, 1}, \ldots, Q_{i, m_{i}} \in\left\{Q_{j}: j \in \mathbf{N}_{0}\right\}$ with $m_{i} \in \mathbf{N}_{0}$ with the following properties.

(c1) $Q_{i, 0}=Q_{i}, Q_{i, m_{i}}=Q_{0}$ for $i \in \mathbf{N}_{0}$.

(c2) $\overline{\kappa_{1} Q_{i, k}} \cap \overline{\kappa_{1} Q_{i, k+1}} \neq \emptyset$ for $i \in \mathbf{N}_{0}$ and $k=0, \ldots, m_{i}-1$.

(c3) There exists a constant $\sigma_{2}=\sigma_{2}\left(\kappa_{1}, \kappa_{2}, N, \alpha, n\right) \geq 1$ such that $Q_{i, k_{1}} \subset \sigma_{2} Q_{i, k_{2}}$ whenever $0 \leq k_{1} \leq k_{2} \leq m_{i}$.

(c4) $Q_{i, k_{1}} \neq Q_{i, k_{2}}$ for $i \in \mathbf{N}_{0}, k_{1}, k_{2} \in\left\{0, \ldots, m_{i}\right\}$ with $k_{1} \neq k_{2}$.

The exact value of $\sigma_{2}$ will be determined below in the construction. Once we have found our $Q_{i, k}$, we define $W_{i, k}:=\frac{3}{2} \kappa_{1} Q_{i, k}$ for $i \in \mathbf{N}_{0}$ and $k=0, \ldots, m_{i}$. It is easy to see that the $W_{i, k}$ and $\mathscr{W}$ satisfy the conditions (C1) and (C2) of Definition 3.5. Note that the balls $B_{i, k}$ satisfying condition (C2) can be chosen, since $\overline{\kappa_{1} Q_{i, k}} \cap \overline{\kappa_{1} Q_{i, k+1}} \neq \emptyset$, $Q_{i, k}$ and $Q_{i, k+1}$ have comparable size, and $W_{i, k}=\frac{3}{2} \kappa_{1} Q_{i, k}$. For a compact subset $K \subset \Omega$ we set $\delta_{0}:=\operatorname{dist}(K, \partial \Omega)>0$. For $W_{i}=\frac{3}{2} \kappa_{1} Q_{i}$ such that $W_{i} \cap K \neq \emptyset$ we easily deduce from the properties of the Whitney covering that $\left|W_{i}\right| \geq c\left(\kappa_{2}\right) \delta_{0}^{n}$. Let $N_{0} \in \mathbf{N}$ be the number of sets $W_{i}$ intersecting $K$. Using the last estimate and (W3) we get

$$
N_{0} c\left(\kappa_{1}\right) \delta_{0}^{n} \leq \sum_{W_{i, k} \cap K \neq \emptyset} \int_{K} \chi_{W_{i, k}} d x \leq N|K|,
$$

which shows (C3). 
Now, let $i \in \mathbf{N}_{0}$. If $i=0$, then it suffices to set $m_{0}:=0$ and $Q_{0,0}:=Q_{0}$. So assume in the following that $i \geq 1$. According to Remark 3.2 there exists a rectifiable path $\gamma_{i}$ which joins $x_{i}$ with $x_{0}$ and $\operatorname{car}(\gamma, \beta) \subset \Omega$. Using this path we inductively construct the finite sequence $Q_{i, 0}, \ldots, Q_{i, m_{i}}$. As a compact subset $\gamma_{i}$ intersects only finitely many $Q_{j}, j \in \mathbf{N}_{0}$. We construct a chain from these $Q_{j}$.

Certainly we set $Q_{i, 0}:=Q_{i}$. Assume that we have constructed the sequence $Q_{i, 0}, \ldots, Q_{i, m}$ satisfying (c2), (c3), and (c4) with $m_{i}$ replaced by $m$. If $Q_{i, m}=Q_{0}$, then we are finished. If $Q_{i, m} \neq Q_{0}$, then we consider

$$
t_{m+1}:=\sup \left\{s: \gamma_{i}(s) \in \kappa_{1} Q_{i, m}\right\} .
$$

Then due to (W1) we can find $Q_{i, m+1} \in\left\{Q_{j}: j \in \mathbf{N}_{0}\right\}$ such that $\gamma_{i}\left(t_{m+1}\right) \in \kappa_{1} Q_{i, m+1}$. By construction we have $\gamma_{i}\left(t_{m+1}\right) \in \overline{\kappa_{1} Q_{i, m}} \cap \overline{\kappa_{1} Q_{i, m+1}}$. So (c2) is satisfied for $k \in$ $0, \ldots, m$. By definition of $t_{k}$ it follows that $Q_{i, m+1} \neq Q_{i, k}$ for $k=0, \ldots, m$. This proves (c4). Only (c3) with $k_{2}=m+1$ requires a real proof. Fix $0 \leq k \leq m+1$. Let $x_{i, k}$ and $x_{i, m+1}$ be the center of $Q_{i, k}$ and $Q_{i, m+1}$, respectively. Let $y \in Q_{i, k}$. Then

$$
\left|x_{i, m+1}-y\right| \leq t_{m+1}+\operatorname{diam} Q_{i, k}+\operatorname{diam} Q_{i, m+1} .
$$

Since $2 \kappa_{1} Q_{i, k} \subset \Omega$, we have

$$
\operatorname{diam}\left(\kappa_{1} Q_{i, k}\right) \leq c \operatorname{dist}\left(\kappa_{1} Q_{i, k}, \partial \Omega\right)
$$

where $c$ only depends on $n$. Note that the sub-path $\gamma_{i}:\left[0, t_{m+1}\right] \rightarrow \Omega$ intersects $\kappa_{1} Q_{i, k}$ and $\kappa_{1} Q_{i, m+1}$, so

$$
\operatorname{dist}\left(\kappa_{1} Q_{i, k}, \partial \Omega\right) \leq t_{m+1}+\operatorname{dist}\left(\kappa_{1} Q_{i, m+1}, \partial \Omega\right) .
$$

Since $\operatorname{car}(\gamma, \beta) \subset \Omega$, we have

$$
t_{m+1} \leq \beta \operatorname{dist}\left(\gamma\left(t_{m+1}\right), \partial \Omega\right) \leq \beta\left(\operatorname{dist}\left(\kappa_{1} Q_{i, m+1}, \partial \Omega\right)+\operatorname{diam}\left(\kappa_{1} Q_{i, m+1}\right)\right) .
$$

Due to (W2) we further have

$$
\operatorname{dist}\left(\kappa_{1} Q_{i, m+1}, \partial \Omega\right) \leq \operatorname{dist}\left(Q_{i, m+1}, \partial \Omega\right) \leq \kappa_{2} \operatorname{diam}\left(Q_{i, m+1}\right) .
$$

Combining the estimates above we get

$$
\left|x_{i, m+1}-y\right| \leq c(1+\beta)\left(1+\kappa_{2}\right) \operatorname{diam}\left(\kappa_{1} Q_{i, m+1}\right)
$$

for all $y \in Q_{i, k}$. This proves

$$
Q_{i, k} \subset c(1+\beta)\left(1+\kappa_{2}\right) 2 \kappa_{1} Q_{i, m+1},
$$

where $c=c(n)$. Since the constant on the right-hand side is independent of $m$ it suffices to choose $\sigma_{2} \geq c(1+\beta)\left(1+\kappa_{2}\right) 2 \kappa_{1}$ in order to ensure $(c 3)$.

Remark 3.10. From the results in [4] and Theorem 3.8 follows that for bounded domains the notions of a John domain, a domain satisfying the Boman chain condition, and a domain satisfying the emanating chain condition coincide.

The treatment of unbounded domains differs only slightly. Different from the bounded case the chains are infinitely long.

Definition 3.11. Let $\Omega \subset \mathbf{R}^{n}$ be an unbounded domain. Further, let $\sigma_{1}, \sigma_{2} \geq 1$. Then we say that $\Omega$ satisfies the emanating chain condition with constants $\sigma_{1}$ and $\sigma_{2}$ if there exists a covering $\mathscr{W}=\left\{W_{i}: i \in \mathbf{N}_{0}\right\}$ of $\Omega$ consisting of open cubes (or balls) such that: 
(C1') We have $\sigma_{1} W \subset \Omega$ for all $W \in \mathscr{W}$ and $\sum_{W \in \mathscr{W}} \chi_{\sigma_{1} W} \leq \sigma_{2} \chi_{\Omega}$ on $\mathbf{R}^{n}$.

(C2') For every $W_{i} \in \mathscr{W}$ there exists a chain of (pairwise different) $W_{i, 0}, W_{i, 1}, W_{i, 2} \ldots$, from $\mathscr{W}$ such that $W_{i, 0}=W_{i}$ and $W_{i, k_{1}} \subset \sigma_{2} W_{i, k_{2}}$ for $0 \leq k_{1} \leq k_{2}$. Moreover, $W_{i, k} \cap W_{i, k+1}, k \geq 0$, contains a ball $B_{i, k}$ such that $W_{i, k} \cup W_{i, k+1} \subset \sigma_{2} B_{i, k}$. The chain $W_{i, 0}, W_{i, 1}, W_{i, 2}, \ldots$ is called chain emanating from $W_{i}$. We define $m_{i}:=\infty$ and call it the length of this chain.

(C3') The set $\left\{i \in \mathbf{N}_{0}: W_{i} \cap K \neq \emptyset\right\}$ is finite for every compact subset $K \subset \Omega$.

The family $\mathscr{W}$ is called the chain-covering of $\Omega$.

Note that the only difference between bounded and unbounded domains is that for bounded domains all chains are finite and end in the central cube $W_{0}$ while for unbounded domains all chains are infinite. For domains $\Omega$ satisfying the emanating chain condition it is easy to see that $\Omega$ is bounded if and only if $|\Omega|<\infty$.

The following theorem shows that unbounded John domains satisfy the emanating chain condition.

Theorem 3.12. Let $\Omega \subset \mathbf{R}^{n}$ be an $\alpha$-John domain with $\Omega \neq \mathbf{R}^{n}$, and let $\left\{Q_{i}\right\}_{i \in \mathbf{N}_{0}}$ be a Whitney covering of $\Omega$ with constants $\kappa_{1}, \kappa_{2}, N$. Let $\sigma_{1}:=\frac{4}{3}$. Define $W_{i}:=\frac{3}{2} \kappa_{1} Q_{i}$ for $i \in \mathbf{N}_{0}$ and $\mathscr{W}:=\left\{W_{i}: i \in \mathbf{N}_{0}\right\}$. Then there exists $\sigma_{2}=$ $\sigma_{2}\left(\kappa_{1}, \kappa_{2}, N, \alpha, n\right) \geq 1$ such that $\Omega$ satisfies the emanating chain condition with constants $\sigma_{1}$ and $\sigma_{2}$ and chain-covering $\mathscr{W}$.

Proof. The case of bounded domains is already covered by Theorem 3.8, so let us assume that $\Omega$ is unbounded. Let $W_{i}:=\frac{3}{2} \kappa_{1} Q_{i}$ for $i \in \mathbf{N}_{0}$ and $\mathscr{W}:=\left\{W_{i}: i \in \mathbf{N}_{0}\right\}$.

For every $i \in \mathbf{N}_{0}$ let $x_{i}$ denote the center of $Q_{i}$. Since $\Omega$ is unbounded, we can find a sub-sequence $\left(i_{j}\right)_{j \in \mathbf{N}}$ such that $\left|x_{i_{j}}\right| \rightarrow \infty$ for $j \rightarrow \infty$.

Fix $i \in \mathbf{N}_{0}$. We construct the chain emanating from $W_{i}$. For every $j \in \mathbf{N}$ with $i \neq$ $i_{j}$, there exists a rectifiable path $\gamma_{i, j}$ from $x_{i}$ to $x_{i_{j}}$ such that $\operatorname{cig}\left(\alpha, \gamma_{i, j}\right) \subset \Omega$. Let $y_{i, j}$ denote the midpoint of $\gamma_{i, j}$ and let $\hat{\gamma}_{i, j}$ denote the first half of $\gamma_{i, j}$. Then $\operatorname{car}\left(\alpha, \hat{\gamma}_{i, j}\right) \subset$ $\Omega$. With the help of the carrot $\operatorname{car}\left(\alpha, \hat{\gamma}_{i, j}\right)$ it is possible to construct exactly as in Theorem 3.8 a (finite) chain $\mathscr{C}_{i, j}$ of cubes from $\mathscr{W}$ which emanates from $W_{i}$ and satisfies $(\mathrm{C} 1)$ and $(\mathrm{C} 2)$ with $W_{0}$ replaced by a cube from $\mathscr{W}$ containing $y_{i, j}$.

We claim that the length of $\mathscr{C}_{i, j}$ goes to infinity as $j \rightarrow \infty$. Indeed, since $\left|x_{i_{j}}\right| \rightarrow \infty$ for $j \rightarrow \infty$, we have $\left|x_{i}-x_{i_{j}}\right| \rightarrow \infty$ and $\left|\gamma_{i, j}\right| \rightarrow \infty$ for $j \rightarrow \infty$. Due to $\operatorname{cig}\left(\alpha, \gamma_{i, j}\right) \subset \Omega$, this implies $\operatorname{dist}\left(y_{i, j}, \partial \Omega\right) \rightarrow \infty$ for $j \rightarrow \infty$. In particular, $\left|x_{i}-y_{i, j}\right| \rightarrow \infty$ for $j \rightarrow \infty$. Since any two neighbours of the Whitney covering have comparable size (see Remark 3.4), two subsequent elements of the chain $\mathscr{C}_{i, j}$ also have comparable size. This and $\left|x_{i}-y_{i, j}\right| \rightarrow \infty$ for $j \rightarrow \infty$ imply that the length of $\mathscr{C}_{i, j}$ goes to infinity as $j \rightarrow \infty$.

Now, we get back to the construction of our chain $W_{i, 0}, W_{i, 1}, W_{i, 2}, \ldots$ Certainly, we define $W_{i, 0}:=W_{i}$. Due to $(\mathrm{C} 1)$ and $(\mathrm{C} 2)$ there is only a finite number of possible choices of $W$ for the second cube in the chains $\mathscr{C}_{i, j}, j \in \mathbf{N}$. So we can pick a subsequence of $\mathscr{C}_{i, j}$ which has always the same cube as the second cube. We define $W_{i, 1}$ to be this cube. By the same argument we can pick a sub-sub-sequence which has always the same cube as the third cube, which defines our cube $W_{i, 2}$. By this recursive procedure we get our sequence $\left(W_{i, k}\right)_{k \in \mathbf{N}_{0}}$ satisfying $\left(\mathrm{C} 1^{\prime}\right)$ and $\left(\mathrm{C} 2^{\prime}\right)$. The proof of condition (C3') is as in the proof of Theorem 3.8. 
Remark 3.13. Note that is irrelevant if we use cubes or balls in Definition 3.5 and 3.11 for our chain-covering $\mathscr{W}$. In fact, it is easy to construct from any chaincovering $\mathscr{W}$ consisting of cubes a different chain-covering $\mathscr{W}^{\prime}$ consisting of balls with corresponding chains. Also the reverse is true. The idea is to replace every cube $W \in$ $\mathscr{W}$ by a finite number of smaller balls with comparable size (the number depends only on $n$ ) which cover this cube $W$. In particular, the definition of the emanating chain condition is independent of the use of cubes or balls. Certainly, by this procedure the constants $\sigma_{1}$ and $\sigma_{2}$ might change.

Remark 3.14. Although the case $\Omega=\mathbf{R}^{n}$ is excluded in Theorem 3.12, it is easy to see that $\mathbf{R}^{n}$ satisfies the emanating chain condition. Indeed, by Theorem 3.12 we see that $\mathbf{R}^{n} \backslash\{0\}$ satisfies the emanating chain condition with a chain-covering $\mathscr{W}$. Now, just add the cube $[-1,1]^{n}$ to the family $\mathscr{W}$ and remove the cubes contained in $[-1 / 2,1 / 2]^{n}$.

Remark 3.15. Let $\Omega \subset \mathbf{R}^{n}$ be a domain satisfying the emanating chain condition with chain-covering $\mathscr{W}$. Then it is possible to choose the balls $B_{i, k}$ in $(\mathrm{C} 2)$ and (C2') from a family $\mathscr{B}$ of balls with

$$
\sum_{B \in \mathscr{B}} \chi_{B} \leq \sigma_{2} \chi_{\Omega} \quad \text { on } \mathbf{R}^{n}
$$

Indeed, let $Y$ denote the set of pairs $\left(W, W^{\prime}\right) \in \mathscr{W} \times \mathscr{W}$ with $W \neq W^{\prime}$ such that there exists a ball $B_{W, W^{\prime}}$ with $B_{W, W^{\prime}} \subset W \cap W^{\prime} \subset \sigma_{2} B_{W, W^{\prime}}$. We can assume that $B_{W, W^{\prime}}=B_{W^{\prime}, W}$ for $\left(W, W^{\prime}\right) \in Y$. Let $\mathscr{B}:=\left\{B_{W, W^{\prime}}:\left(W, W^{\prime}\right) \in Y\right\}$. Then by $(\mathrm{C} 1)$ follows

$$
\sum_{B \in \mathscr{B}} \chi_{B} \leq \sigma_{2} \chi_{\Omega}
$$

Now, it suffices to choose the $B_{i, k}$ in $(\mathrm{C} 2)$ and $\left(\mathrm{C} 2{ }^{\prime}\right)$ from the family $\mathscr{B}$.

Remark 3.16. Iwaniec and Nolder define in [20] also the Boman chain condition for unbounded domains. They say that an unbounded domain $\Omega \subset \mathbf{R}^{n}$ satisfies the Boman chain condition with constants $\sigma_{1}$ and $\sigma_{2}$ if it can be written as the countable union of bounded domains $\Omega_{i}$ with $\Omega_{1} \subset \Omega_{2} \subset \ldots$ which satisfy the Boman chain condition with constants $\sigma_{1}$ and $\sigma_{2}$. Note that the same construction can be used to characterize unbounded John domains starting from bounded ones. As a consequence of the characterization "John = Boman" for bounded domains, see Buckley, Koskela, and $\mathrm{Lu} \mathrm{[4],} \mathrm{we} \mathrm{get} \mathrm{that} \mathrm{"John} \mathrm{=} \mathrm{Boman"} \mathrm{also} \mathrm{for} \mathrm{unbounded} \mathrm{domains.}$

Remark 3.17. The half space $\left\{\left(x_{1}, \ldots, x_{n}\right) \in \mathbf{R}^{n}: x_{n}>0\right\}$, the whole space $\mathbf{R}^{n}$ and exterior Lipschitz domains are unbounded John domains, which satisfy the Boman chain condition and the emanating chain condition.

We have seen in Theorem 3.12 that every unbounded John domain also satisfies the emanating chain condition. However, the converse is not true. Indeed, the aperture domain

$$
\left\{\left(x_{1}, x_{2}\right) \in \mathbf{R}^{2}: x_{2} \neq 0 \text { or }\left|x_{1}\right|<1\right\}
$$

and domains with (at least two) conical outlets (cf. [25]) satisfy the emanating chain condition but are no John domain. 


\section{Decomposition of $L_{w, 0}^{q}(\Omega)$}

In the following let $\Omega \subset \mathbf{R}^{n}$ be a domain satisfying the emanating chain condition. Let $w \in A_{q}$. For the definition of the space $L_{w, 0}^{q}(\Omega)$ we distinguish the cases, when $\Omega$ is bounded and when $\Omega$ is unbounded.

If $\Omega$ is bounded, $1 \leq q<\infty$, and $w \in A_{q}$, then we define

$$
L_{w, 0}^{q}(\Omega):=\left\{f \in L_{w}^{q}(\Omega): \int_{\Omega} f d x=0\right\} .
$$

If $\Omega$ is unbounded, $1<q<\infty$, and $w \in A_{q}$, then we define

$$
L_{w, 0}^{q}(\Omega):=L_{w}^{q}(\Omega) .
$$

We equip $L_{w, 0}^{q}(\Omega)$ with the norm of $L_{w}^{q}(\Omega)$.

Remark 4.1. It can be shown that if $1<q<\infty$ and $w \in A_{q}$, then $L_{w, 0}^{q}(\Omega)$ is the closure of $C_{0,0}^{\infty}(\Omega)$ for bounded and unbounded $\Omega$.

Our main result of this section is the following decomposition result.

Theorem 4.2. (Decomposition Theorem) Let $\Omega \subset \mathbf{R}^{n}$ be a domain satisfying the emanating chain condition with constants $\sigma_{1}, \sigma_{2}$ and chain-covering $\mathscr{W}=$ $\left\{W_{i}: i \in \mathbf{N}_{0}\right\}$. Then there exists a family of linear operators $T_{i}: C_{0,0}^{\infty}(\Omega) \rightarrow C_{0,0}^{\infty}\left(W_{i}\right)$, $i \in \mathbf{N}_{0}$ such that for all $1<q<\infty$ and all $w \in A_{q}$ the following holds:

(a) For each $i \in \mathbf{N}_{0}$ the operator $T_{i}$ maps $L_{w, 0}^{q}(\Omega)$ continuously into $L_{w, 0}^{q}\left(W_{i}\right)$.

(b) For each $i \in \mathbf{N}_{0}$ and all $f \in L_{w, 0}^{q}(\Omega)$ holds

$$
\left|T_{i} f\right| \leq c \sigma_{2} \chi_{W_{i}} M f \quad \text { almost everywhere. }
$$

(c) The family $T_{i} f$ is a decomposition of $f$ in $L_{w, 0}^{q}(\Omega)$, i.e.

$$
f=\sum_{i \geq 0} T_{i} f \quad \text { in } L_{w, 0}^{q}(\Omega)
$$

for all $f \in L_{w, 0}^{q}(\Omega)$. The convergence is unconditionally, i.e. every permutation of the series converges.

(d) The mapping $f \mapsto\left\|T_{i} f\right\|_{L_{w, 0}^{q}\left(W_{i}\right)}$ from $L_{w, 0}^{q}(\Omega)$ into $l^{q}\left(\mathbf{N}_{0}\right)$ is bounded and

$$
\frac{1}{c}\|f\|_{L_{w, 0}^{q}(\Omega)} \leq\left(\sum_{i \geq 0}\left\|T_{i} f\right\|_{L_{w, 0}^{q}\left(W_{i}\right)}^{q}\right)^{\frac{1}{q}} \leq c\|f\|_{L_{w, 0}^{q}(\Omega)}
$$

with $c=c\left(\sigma_{1}, \sigma_{2}, q, A_{q}(w)\right)$.

(e) If $\Omega$ is bounded and $f \in C_{0,0}^{\infty}(\Omega)$, then $\left\{i \geq 0: T_{i} f \neq 0\right\}$ is finite.

Proof. We treat the case that the domain $\Omega$ is bounded and the case that it is unbounded simultaneously. In the case of an unbounded domain we use the convention that in the arguments below the conditions (C1) and (C2) has to be replaced by $\left(\mathrm{C} 1^{\prime}\right)$ and $(\mathrm{C} 2 ')$. Recall that $m_{i}=\infty$ for unbounded domains.

In the following let $1<q<\infty$ and $w \in A_{q}$. We begin with the construction of our operators $T_{i}$. Let $f \in C_{0,0}^{\infty}(\Omega)$. Due to $(\mathrm{C} 1)$ and $\Omega=\bigcup_{i>0} W_{i}$, there exists a smooth partition of unity $\left\{\xi_{i}\right\}_{i \geq 0}$ subordinate to the covering $\left\{W_{i}\right\}_{i \geq 0}$, cf. [2, Theorem 3.14]. For $f \in L_{\text {loc }}^{1}(\Omega)$ we define $S_{i} f:=\xi_{i} f$ for all $i \in \mathbf{N}_{0}$. Then the $S_{i}$ are linear maps from $L_{\text {loc }}^{1}(\Omega)$ to $L^{1}\left(W_{i}\right)$ and from $C_{0}^{\infty}(\Omega)$ to $C_{0}^{\infty}\left(W_{i}\right)$. Moreover, $\left|S_{i} f\right| \leq \chi_{W_{i}}|f|$ and $\sum_{i \geq 0} S_{i} f=f$ almost everywhere, and if $f \in L_{w}^{q}(\Omega)$, then $\sum_{i \geq 0} S_{i} f=f$ in $L_{w}^{q}(\Omega)$ by 
the dominated convergence theorem. We have not finished our proof yet, since $S_{i} f$ in general does not satisfy $\int S_{i} f d x=0$.

Let $W_{i, k}$ and $B_{i, k}$ be as in $(\mathrm{C} 2)$. Due to Remark 3.15 we can assume that all these $B_{i, k}$ are from a family $\mathscr{B}$ of balls which satisfies

$$
\sum_{B \in \mathscr{B}} \chi_{B} \leq \sigma_{2} \chi_{\Omega} \quad \text { on } \mathbf{R}^{n}
$$

For every $B \in \mathscr{B}$ let $\eta_{B} \in C_{0}^{\infty}(B)$ with $\eta_{B} \geq 0, \int \eta_{B} d x=1$, and $\left\|\eta_{B}\right\|_{\infty} \leq c /|B|$, where $c=c(n)$. For $B_{i, k} \in \mathscr{B}$ we define $\eta_{i, k}:=\eta_{B_{i, k}}$. From the properties of $B_{i, k}$ follows

$$
\begin{aligned}
\eta_{i, k} & \in C_{0}^{\infty}\left(W_{i, k}\right) \cap C_{0}^{\infty}\left(W_{i, k+1}\right), \\
\left\|\eta_{i, k}\right\|_{\infty} & \leq c \min \left\{\frac{1}{\left|W_{i, k}\right|}, \frac{1}{\left|W_{i, k+1}\right|}\right\}
\end{aligned}
$$

for all $i, k \geq 0$ with $0 \leq k \leq m_{i}-1$.

In the case of a bounded domain $\Omega$ we pick a function $\eta_{0} \in C^{\infty}\left(W_{0}\right)$ with $\eta_{0} \geq 0$, $\int \eta_{0} d x=1$ and $\left\|\eta_{0}\right\|_{\infty} \leq c /\left|W_{0}\right|$, where $c=c(n)$. Then we define $\eta_{i, m_{i}}:=\eta_{0}$ for every $i \geq 0$. In particular, we have

$$
\begin{aligned}
\eta_{i, m_{i}} & \in C_{0}^{\infty}\left(W_{i, m_{i}}\right), \\
\left\|\eta_{i, m_{i}}\right\|_{\infty} & \leq \frac{c}{\left|W_{i, m_{i}}\right|}
\end{aligned}
$$

for all $i \geq 0$.

We define $T_{i} f$ for all $f \in C_{0,0}^{\infty}(\Omega)$ and all $f \in L_{w, 0}^{q}(\Omega)$ by

$$
T_{i} f:=S_{i} f-\int_{W_{i}} S_{i} f d x \eta_{i, 0}+\sum_{\substack{j \geq 0 \\ j \neq i}}\left(\int_{W_{j}} S_{j} f d x \sum_{\substack{k: 0<k \leq m_{j} \\ W_{j, k}=W_{i}}}\left(\eta_{j, k-1}-\eta_{j, k}\right)\right) .
$$

The sum over $j$ could be restricted to all $j$ such that $W_{i}$ is contained in the chain emanating from $W_{j}$, since for all other $j$ the sum over $k$ is empty. Note that the sum over $k$ consists of at most one summand, since all cubes in a chain are pairwise different. Since the sum over $j$ may still be countable, it is not clear if $T_{i} f$ is well defined by (4.9). Therefore, we show now that the sum on the right-hand side of (4.9) converges almost everywhere, absolutely and can be estimated in terms of the maximal function $M f$. From this follows immediately that $T_{i} f$ is well defined for all $f \in C_{0,0}^{\infty}(\Omega)$ and all $f \in L_{w, 0}^{q}(\Omega)$. We define pointwise

$$
U_{i} f:=\sum_{\substack{j \geq 0 \\ j \neq i}}\left(\left|\int_{W_{j}} S_{j} f d x\right| \sum_{\substack{k: 0<k \leq m_{j} \\ W_{j, k}=W_{i}}}\left(\eta_{j, k-1}+\eta_{j, k}\right)\right),
$$

which is well defined but may be infinite. Now, fix $i, j, k \geq 0$ with $0<k \leq m_{j}$ be such that $W_{i}=W_{j, k}$. Together with (C2) we have

$$
W_{j}=W_{j, 0} \subset \sigma_{2} W_{j, k}=\sigma_{2} W_{i} .
$$

Moreover, the choice of $\eta_{j, k}$ and $\eta_{j, k+1}$, see (4.7) and (4.8), imply

$$
\eta_{j, k-1}+\eta_{j, k} \leq \frac{c \chi_{W_{j, k}}}{\left|W_{j, k}\right|}=\frac{c \chi_{W_{i}}}{\left|W_{i}\right|} .
$$


Also the summation over $k$ in (4.10) contains at most one summand. So we get almost everywhere

$$
\sum_{\substack{k: 0<k \leq m_{j} \\ W_{j, k}=W_{i}}}\left(\eta_{j, k-1}+\eta_{j, k}\right) \leq \frac{c \chi_{W_{i}}}{\left|W_{i}\right|}
$$

With $\left|S_{j} f\right| \leq|f|,(4.11)$, and (C1) we conclude

$$
U_{i} f \leq \sum_{\substack{j \geq 0 \\ j \neq i}} \int_{W_{j}}|f| d x \frac{c \chi_{W_{i}}}{\left|W_{i}\right|} \leq c \sigma_{2} \int_{\sigma_{2} W_{i}}|f| d x \frac{\chi_{W_{i}}}{\left|W_{i}\right|} \leq c \sigma_{2} \chi_{W_{i}} M f .
$$

Analogously, we estimate

$$
\begin{aligned}
\left|\int_{W_{i}} S_{i} f d x\right| \eta_{i, 0} & \leq c \chi_{W_{i}} M f \\
\left|S_{i} f\right| & \leq \chi_{W_{i}}|f| \leq \chi_{W_{i}} M f
\end{aligned}
$$

almost everywhere. With (C1), (4.13), and (4.14) we can conclude that the sum in the definition of $T_{i}$, see (4.9), converges almost everywhere, absolutely and

$$
\left|T_{i} f\right| \leq c \sigma_{2} \chi_{W_{i}} M f \quad \text { almost everywhere. }
$$

This proves (b). Now, Theorem 2.4 shows that the linear operators $T_{i}: L_{w, 0}^{q}(\Omega) \rightarrow$ $L_{w, 0}^{q}\left(W_{i}\right)$ are bounded.

We now prove (a). It remains to show that $\int T_{i} f d x=0$ for every $f \in L_{w, 0}^{q}(\Omega)$. We have shown above that the sum in the definition of $T_{i}$, see (4.9), converges almost everywhere absolutely. This, (4.15), Theorem 2.4, and the dominated convergence theorem imply that the sum in the definition of $T_{i}$ also converges in $L_{w}^{q}\left(W_{i}\right)$. Since $w \in A_{q}$ we have $L_{w}^{q}\left(W_{i}\right) \hookrightarrow L^{1}\left(W_{i}\right)$, and thus the sum in the definition of $T_{i}$ also converges in $L^{1}\left(W_{i}\right)$. This, $\int \eta_{j, k} d x=1$ for every $j, k \geq 0$ with $0 \leq k \leq m_{j}$, and (4.9) imply

$$
\int T_{i} f d x=\int S_{i} f d x-\int S_{i} f d x=0,
$$

which proves (a). It also shows that $T_{i}: C_{0}^{\infty}(\Omega) \rightarrow L_{0}^{1}\left(W_{i}\right)$, since $L_{w, 0}^{q}\left(W_{i}\right) \hookrightarrow L_{0}^{1}\left(W_{i}\right)$.

Let $f \in C_{0,0}^{\infty}(\Omega)$ and fix $i \geq 0$. Since the balls $B_{i, k}$ with $i, k \in \mathbf{N}_{0}, 0 \leq k<m_{i}$, are all from the family $\mathscr{B}$, it follows from (4.6), and the construction of the $\eta_{i, k}$ that only finitely many different functions $\eta_{B}$ with $B \in \mathscr{B}$ appear in the definition of $T_{i} f$, see (4.9). All of these functions are from $C_{0,0}^{\infty}\left(W_{i}\right)$. Since we can resort the sum in the definition of $T_{i} f$ arbitrarily (see above), $T_{i} f$ can be written as the linear combination of finitely many $C_{0,0}^{\infty}\left(W_{i}\right)$-functions. This proves $T_{i} f \in C_{0,0}^{\infty}\left(W_{i}\right)$, so with (4.16) we get $T_{i}: C_{0,0}^{\infty}(\Omega) \rightarrow C_{0,0}^{\infty}\left(W_{i}\right)$.

Now, we prove that

$$
\sum_{i \geq 0} T_{i} f=f \quad \text { almost everywhere }
$$

for all $f \in L_{w, 0}^{q}(\Omega)$. Let us fix $f \in L_{w, 0}^{q}(\Omega)$ and $i_{0} \in \mathbf{N}_{0}$. We show that (4.17) holds on $W_{i_{0}}$. Due to (C1), the sum on the left-hand side of (4.17) involves on $W_{i_{0}}$ only finitely many summands. Moreover, we have shown above that the sum in the definition of $T_{i}$, see (4.9), converges almost everywhere absolutely. This allows us 
in the following calculations to resort the sums as we like. On $W_{i_{0}}$ we calculate pointwise

$$
\sum_{i \geq 0} T_{i} f=\sum_{i \geq 0}\left[S_{i} f-\int_{W_{i}} S_{i} f d x \eta_{i, 0} \sum_{i \geq 0}\left(+\sum_{\substack{j \geq 0 \\ j \neq i}}\left(\int_{W_{j}} S_{j} f d x \sum_{\substack{k: 0<k \leq m_{j} \\ W_{j, k}=W_{i}}}\left(\eta_{j, k-1}-\eta_{j, k}\right)\right)\right] .\right.
$$

With $\sum_{i \geq 0} S_{i} f=f$ we get pointwise on $W_{i_{0}}$

$$
\begin{aligned}
& \sum_{i \geq 0} T_{i} f=f-\sum_{i \geq 0}\left(\int_{W_{i}} S_{i} f d x \eta_{i, 0}\right) \\
& +\sum_{j \geq 0}\left[\int_{W_{j}} S_{j} f d x \sum_{k: 0<k \leq m_{j}}\left(\left(\eta_{j, k-1}-\eta_{j, k}\right) \sum_{\substack{i \geq 0 \\
i \neq j \\
W_{i}=W_{j, k}}} 1\right)\right] .
\end{aligned}
$$

Let us consider the last sum over $i$. For this, we fix $j, k \geq 0$ with $0<k \leq m_{j}$. Then there exists a unique $i \geq 0$ such that $W_{j, k}=W_{i}$. Since $k>0$, we have by (C2) that $W_{i}=W_{j, k} \neq W_{j, 0}=W_{j}$, which implies $i \neq j$. Therefore, we have

$$
\sum_{\substack{i \geq 0 \\ i \neq j \\ W_{i}=W_{j, k}}} 1=1
$$

for all $j, k \geq 0$ with $0<k \leq m_{j}$. This and (4.18) prove pointwise on $W_{i_{0}}$

$$
\begin{aligned}
\sum_{i \geq 0} T_{i} f= & f-\sum_{i \geq 0}\left(\int_{W_{i}} S_{i} f d x \eta_{i, 0}\right) \\
& +\sum_{j \geq 0}\left[\int_{W_{i}} S_{j} f d x \sum_{k: 0<k \leq m_{j}}\left(\eta_{j, k-1}-\eta_{j, k}\right)\right] \\
= & f-\sum_{i \geq 0}\left(\int_{W_{i}} S_{i} f d x \eta_{i, 0}\right)+\sum_{j \geq 0}\left[\int_{W_{j}} S_{j} f d x\left(\eta_{j, 0}-\eta_{j, m_{j}}\right)\right] \\
= & f-\sum_{j \geq 0}\left(\int_{W_{j}} S_{j} f d x \eta_{j, m_{j}}\right) .
\end{aligned}
$$

Note that for unbounded $\Omega$ the terms involving $\eta_{j, m_{j}}$ do not appear, which proves (4.17) in this case. Let us continue with the case of a bounded domain $\Omega$. In that case we have $\eta_{j, m_{j}}=\eta_{0}$, so pointwise on $W_{i_{0}}$

$$
\sum_{i \geq 0} T_{i} f=f-\eta_{0} \sum_{j \geq 0} \int_{W_{j}} S_{j} f d x
$$

Since $\sum_{j \geq 0} S_{j} f=f$ in $L_{w}^{q}(\Omega)$ and $L_{w}^{q}(\Omega) \hookrightarrow L^{1}(\Omega)$, we have $\sum_{j \geq 0} S_{j} f=f$ in $L^{1}(\Omega)$. Thus, $\sum_{j \geq 0} \int_{W_{j}} S_{j} f d x=\int_{\Omega} f d x=0$. This and (4.20) prove (4.17) on $W_{i_{0}}$ and therefore on $\Omega$.

Let us show the unconditional convergence of $\sum_{i \geq 0} T_{i} f$ in $L_{w, 0}^{q}(\Omega)$ for $f \in$ $L_{w, 0}^{q}(\Omega)$. We have to show that for every permutation $\sigma$ of $\mathbf{N}_{0}$ the sum $\sum_{i \geq 0} T_{\sigma(i)} f$ 
converges to $f$. With (4.17), (b) and (C2) we estimate

$$
\left\|f-\sum_{i=0}^{k} T_{\sigma(i)} f\right\|_{L_{w, 0}^{q}(\Omega)} \leq c\left\|\sum_{i>k} \chi_{W_{\sigma(i)}} M f\right\|_{L_{w}^{q}(\Omega)} .
$$

Due to $(\mathrm{C} 1)$ we have $\sum_{i>k} \chi_{W_{\sigma(i)}} \leq N \chi_{\Omega}$ and therefore $\sum_{i>k} \chi_{W_{\sigma(i)}} \rightarrow 0$ almost everywhere for $k \rightarrow \infty$. So by Theorem 2.4 and the dominated convergence theorem the right-hand side of (4.21) converges to zero. This proves the unconditional convergence of $\sum_{i>0} T_{i} f$ in $L_{w, 0}^{q}(\Omega)$. We have proved (c).

For $f \in L_{w}^{q}(\Omega)$ we estimate with (b) and (C1)

$$
\begin{aligned}
\left(\sum_{i \geq 0}\left\|T_{i} f\right\|_{L_{w, 0}^{q}\left(W_{i}\right)}^{q}\right)^{\frac{1}{q}} & \leq c\left(\sum_{i \geq 0}\left\|\chi_{W_{i}} M f\right\|_{L_{w}^{q}\left(W_{i}\right)}^{q}\right)^{\frac{1}{q}} \\
& \leq c\left(\int_{\Omega} \sum_{i \geq 0} \chi_{W_{i}}(M f)^{q} w d x\right)^{\frac{1}{q}} \\
& \leq c\|M f\|_{L_{w}^{q}(\Omega)} \\
& \leq c\|f\|_{L_{w, 0}^{q}(\Omega)}
\end{aligned}
$$

This proves the second part of (4.5). On the other hand with (c) and (C1) we get

$$
\|f\|_{L_{w, 0}^{q}(\Omega)}=\left\|\sum_{i \geq 0} T_{i} f\right\|_{L_{w, 0}^{q}(\Omega)} \leq c\left(\sum_{i \geq 0}\left\|T_{i} f\right\|_{L_{w, 0}^{q}\left(W_{i}\right)}^{q}\right)^{\frac{1}{q}} .
$$

This proves $(\mathrm{d})$.

Let us prove finally (d). Thus, let $\Omega$ be bounded and $f \in C_{0,0}^{\infty}(\Omega)$. We have to show that $J:=\left\{i \geq 0: T_{i} f \neq 0\right\}$ is finite. Define $G:=\left\{i \geq 0: S_{i} f \neq 0\right\}$. Since $f \in C_{0,0}^{\infty}(\Omega)$, it follows by (C3) that $G$ is a finite set. Since $\Omega$ is bounded, every chain emanating from $W_{j}$ with $j \in G$ has finite length. In particular, the collection of chains emanating from some $W_{j}$ with $j \in G$ passes only through a finite number of cubes. Let $H$ denote the indices of all these cubes. Now, it is easy to see that $T_{i} f=0$ for every $i \in \mathbf{N}_{0} \backslash(G \cup H)$. Indeed, for $i \in \mathbf{N}_{0} \backslash G$ the first two terms in the definition of $T_{i} f$, see (4.9), are zero. For $i \in \mathbf{N}_{0} \backslash H$ the last term in the definition of $T_{i} f$ is zero, since $j \in G$ or the sum over $k$ is empty. Since $G$ and $H$ are finite sets, we have $|J| \leq|G \cup H|<\infty$. So (e) holds.

Lemma 4.22. Let $\Omega \subset \mathbf{R}^{n}$ be a bounded domain satisfying the emanating chain condition with constants $\sigma_{1}, \sigma_{2}$, let $1<q<\infty$, and $w \in A_{q}$. Let $\eta \in L^{\infty}(\Omega)$ with $\int_{\Omega} \eta d x=1$. Then $U_{\eta}: f \mapsto f-\eta \int_{\Omega} f d x$ and $V_{\eta}: f \mapsto \eta \int_{\Omega} f d x$ are a bounded, linear mappings from $L_{w}^{q}(\Omega)$ to $L_{w, 0}^{q}(\Omega)$ and from $L_{w}^{q}(\Omega)$ to $L_{w}^{q}(\Omega)$, respectively. The mapping $U_{\eta}$ is onto and

$$
\begin{array}{r}
\left\|f-\eta \int_{\Omega} f d x\right\|_{L_{w, 0}^{q}(\Omega)} \leq c\|f\|_{L_{w}^{q}(\Omega)}, \\
\left\|\eta \int_{\Omega} f d x\right\|_{L_{w, 0}^{q}(\Omega)} \leq c\|f\|_{L_{w}^{q}(\Omega)},
\end{array}
$$


for all $f \in L_{w}^{q}(\Omega)$. The constants depend only on $q, A_{q}(w), \sigma_{2}$, and $\|\eta\|_{\infty}|\Omega|$. If $\eta \in C_{0}^{\infty}(\Omega)$, then $U_{\eta}: C_{0}^{\infty}(\Omega) \rightarrow C_{0,0}^{\infty}(\Omega)$.

Proof. Let $W_{0}$ denote the central cube of $\Omega$. Then with Hölder's inequality and $\Omega \subset \sigma_{2} W_{0}$ (see Remark 3.6)

$$
\begin{aligned}
\left\|\eta \int_{\Omega} f d x\right\|_{L_{w}^{q}(\Omega)} & \leq \frac{|\Omega|}{\left|W_{0}\right|}\|\eta\|_{\infty}\left\|\chi_{\Omega}\right\|_{L_{w}^{q}\left(\mathbf{R}^{n}\right)}\|f\|_{L_{w}^{q}(\Omega)}\left\|\chi_{\Omega}\right\|_{L_{w^{\prime}}^{q^{\prime}\left(\mathbf{R}^{n}\right)}} \\
& \leq \sigma_{2}^{n} \frac{\left\|\chi_{\sigma_{2} W_{0}}\right\|_{L_{w}^{q}\left(\mathbf{R}^{n}\right)}\left\|\chi_{\sigma_{2} W_{0}}\right\|_{L_{w^{\prime}}^{q^{\prime}\left(\mathbf{R}^{n}\right)}}\|\eta\|_{\infty}|\Omega|\|f\|_{L_{w}^{q}(\Omega)}}{\left|\sigma_{2} W_{0}\right|} \\
& \leq \sigma_{2}^{n}\left(A_{q}(w)\right)^{\frac{1}{q}}\|\eta\|_{\infty}|\Omega|\|f\|_{L_{w}^{q}(\Omega)},
\end{aligned}
$$

where we have used $w \in A_{q}$ in the last step. This proves (4.23). The mapping $U_{\eta}$ is onto, since it is the identity on $L_{w, 0}^{q}(\Omega)$. Since $\int \eta d x=1$, we have $\int_{\Omega} U_{\eta} f d x=0$. That $U_{\eta}$ maps $C_{0}^{\infty}(\Omega)$ into $C_{0,0}^{\infty}(\Omega)$ for $\eta \in C_{0}^{\infty}(\Omega)$ is obvious.

Remark 4.24. If $\Omega \subset \mathbf{R}^{n}$ is a bounded domain satisfying the emanating chain condition, $1<q<\infty$, and $w \in A_{q}$, then we can combine Lemma 4.22 with Theorem 4.2 to extend our operators $T_{i}: L_{w, 0}^{q}(\Omega) \rightarrow L_{w, 0}^{q}\left(W_{i}\right)$ to $\hat{T}_{i}: L_{w}^{q}(\Omega) \rightarrow L_{w, 0}^{q}\left(W_{i}\right)$ for $i \geq 0$. Indeed, let $\eta$ be as in Lemma 4.22 , then the operators $\hat{T}_{i}:=T_{i} \circ U_{\eta}$ have the desired property. Moreover,

$$
\sum_{i \geq 0} \hat{T}_{i} f=\sum_{i \geq 0} T_{i}\left(U_{\eta} f\right)=U_{\eta} f=f-\eta \int_{\Omega} f d x
$$

with unconditional convergence in $L_{w, 0}^{q}(\Omega)$. It is easily seen that the operators $\hat{T}_{i}$ satisfy (a), (b), and (d) of Theorem 4.2.

If additionally $\eta \in C_{0}^{\infty}(\Omega)$, then $\hat{T}_{i}: C_{0}^{\infty}(\Omega) \rightarrow C_{0,0}^{\infty}(\Omega)$ and the $\hat{T}_{i}$ also satisfy (e) of Theorem 4.2.

The following results will also be useful for the applications.

Lemma 4.25. Let $\Omega \subset \mathbf{R}^{n}$ be a bounded domain satisfying the emanating chain condition, $1<q<\infty$, and $w \in A_{q}$. Further let $\eta \in L^{\infty}(\Omega)$ with $\int_{\Omega} \eta d x=1$. Then

$$
\|f\|_{L_{w}^{q}(\Omega)} \leq c\left\|f-\langle f\rangle_{\Omega}\right\|_{L_{w, 0}^{q}(\Omega)}+|\langle f, \eta\rangle|\left\|\chi_{\Omega}\right\|_{L_{w}^{q}(\Omega)}
$$

for all $f \in L_{w}^{q}(\Omega)$. The constants depend only on $q, A_{q}(w), \sigma_{2}$, and $\|\eta\|_{\infty}|\Omega|$.

Proof. For $f \in L_{w}^{q}(\Omega)$ we estimate

$$
\|f\|_{L_{w}^{q}(\Omega)}=\sup _{\|g\|_{L_{w^{\prime}}^{q^{\prime}}(\Omega)} \leq 1}\langle f, g\rangle=\sup _{\|g\|_{L_{w^{\prime}}^{q^{\prime}}(\Omega)} \leq 1}\left(\left\langle f, U_{\eta} g\right\rangle+\langle f, \eta\rangle \int_{\Omega} g d y\right),
$$

where $U_{\eta} g=g-\eta \int_{\Omega} g d y \in L_{w^{\prime}, 0}^{q^{\prime}}(\Omega)$. With Lemma 4.22 we get

$$
\begin{aligned}
\|f\|_{L_{w}^{q}(\Omega)} & \leq c \sup _{\|h\|_{L_{w^{\prime}, 0}^{q^{\prime}}(\Omega)} \leq 1}\langle f, h\rangle+\sup _{\|g\|_{L_{w^{\prime}}^{q^{\prime}}}(\Omega)}\left\langle f(\eta, \eta\rangle \int_{\Omega} g d y\right. \\
& \leq c\left\|f-\langle f\rangle_{\Omega}\right\|_{L_{w, 0}^{q}(\Omega)}+|\langle f, \eta\rangle|\left\|\chi_{\Omega}\right\|_{L_{w}^{q}(\Omega)} .
\end{aligned}
$$

This proves the claim. 


\section{Applications}

In this section we show how our Decomposition Theorem 4.2 can be used to generalize several results known for cubes or balls to domains satisfying the emanating chain condition.

5.1. Poincaré's inequality. First, we give a simple proof of Poincaré's inequality for bounded domains which satisfy the emanating chain condition using our Decomposition Theorem 4.2. The result has already been proved by Chua in [5] using the Boman chain condition. More general cases have been considered in [18].

Theorem 5.1. Let $\Omega \subset \mathbf{R}^{n}$ be a bounded domain satisfying the emanating chain condition with constants $\sigma_{1}$ and $\sigma_{2}$. Then for all $f \in W_{w}^{1, q}(\Omega)$ holds

$$
\left\|f-\langle f\rangle_{\Omega}\right\|_{L_{w}^{q}(\Omega)} \leq c \operatorname{diam}(\Omega)\|\nabla f\|_{L_{w}^{q}(\Omega)} .
$$

The constant only depends on $\sigma_{1}, \sigma_{2}, q$, and $A_{q}(w)$.

Proof. Let $f \in L_{w}^{q}(\Omega)$. Then with Theorem 4.2 (using balls for $W_{i}$ ) follows

$$
\begin{aligned}
\left\|f-\langle f\rangle_{\Omega}\right\|_{L_{w, 0}^{q}(\Omega)} & =\sup _{\|g\|_{L_{w^{\prime}, 0}^{q^{\prime}(\Omega)}} \leq 1}\langle f, g\rangle \\
& =\sup _{\|g\|_{L_{w^{\prime}, 0}^{q^{\prime}}(\Omega)} \leq 1} \sum_{i \geq 0}\left\langle f, T_{i} g\right\rangle \\
& =\sup _{\|g\|_{L_{w^{\prime}, 0}^{q^{\prime}}(\Omega)}} \sum_{i \geq 0}\left\langle f-\langle f\rangle_{W_{i}}, T_{i} g\right\rangle . \\
& \leq \sup _{\|g\|_{L_{w^{\prime}, 0}^{q^{\prime}}} \leq 1} \sum_{i \geq 0}\left\|f-\langle f\rangle_{W_{i}}\right\|_{L_{w}^{q}\left(W_{i}\right)}\left\|T_{i} g\right\|_{L_{w^{\prime}, 0}^{q^{\prime}}(\Omega)} .
\end{aligned}
$$

It has been proven in [12, Theorem 1.5] that Poincaré's inequality holds for balls, i.e.

$$
\left\|f-\langle f\rangle_{W_{i}}\right\|_{L_{w}^{q}\left(W_{i}\right)} \leq c \operatorname{diam}\left(W_{i}\right)\|\nabla f\|_{L_{w}^{q}\left(W_{i}\right)}
$$

where $c$ depends only on $q$ and $A_{q}(w)$. This, $\operatorname{diam}\left(W_{i}\right) \leq \operatorname{diam}(\Omega)$, Hölder's inequality, (C1), and Theorem 4.2 imply

$$
\begin{aligned}
\left\|f-\langle f\rangle_{\Omega}\right\|_{L_{w}^{q}(\Omega)} & \leq c \operatorname{diam}(\Omega) \sup _{\|g\|_{L^{q^{\prime}, 0}}(\Omega)} \sum_{i \geq 0}\|\nabla f\|_{L_{w}^{q}\left(W_{i}\right)}\left\|T_{i} g\right\|_{L_{w^{\prime}, 0}^{q^{\prime}}(\Omega)} \\
& \leq c \operatorname{diam}(\Omega) \sup _{\|g\|_{L_{w^{\prime}, 0}^{q^{\prime}}} \leq 1}\left(\sum_{i \geq 0}\|\nabla f\|_{L_{w}^{q}\left(W_{i}\right)}^{q}\right)^{\frac{1}{q}}\left(\sum_{i \geq 0}\left\|T_{i} g\right\|_{L_{w^{\prime}, 0}^{q^{q^{\prime}}}(\Omega)}^{q^{\prime}}\right)^{\frac{1}{q^{\prime}}} \\
& \leq c \operatorname{diam}(\Omega) \sup _{\|g\|_{L_{w^{\prime}, 0}^{q^{\prime}}(\Omega)} \leq 1}\|\nabla f\|_{L_{w}^{q}(\Omega)}\|g\|_{L_{w^{\prime}, 0}^{q^{\prime}}(\Omega)} \\
& \leq c \operatorname{diam}(\Omega)\|\nabla f\|_{L_{w}^{q}(\Omega)} .
\end{aligned}
$$

This proves the claim.

5.2. The divergence equation, negative norm theorem, and Korn's inequality. For a domain $\Omega \subset \mathbf{R}^{n}$ and $1<q<\infty$ we define the weighted homogeneous Sobolev space $D_{w, 0}^{1, q}(\Omega)$ to be the completion of $C_{0}^{\infty}(\Omega)$ with respect to the gradient norm $\|\nabla \cdot\|_{L_{w}^{q}(\Omega)}$. Then $D_{0}^{1, q}(\Omega)$ is a Banach space. Note that if $\Omega$ is 
bounded, then $D_{w, 0}^{1, q}(\Omega)=W_{w, 0}^{1, q}(\Omega)$. We refer to [29] for more details on homogeneous Sobolev spaces.

Theorem 5.2. Let $\Omega \subset \mathbf{R}^{n}$ be a domain satisfying the emanating chain condition with constants $\sigma_{1}$ and $\sigma_{2}$. Then there exists a linear operator B: $C_{0}^{\infty}(\Omega) \rightarrow$ $\left(L_{\text {loc }}^{1}(\Omega)\right)^{n}$ which for all $1<q<\infty$ and all $w \in A_{q}$ extends uniquely to an operator $\mathrm{B}: L_{w, 0}^{q}(\Omega) \rightarrow\left(D_{w, 0}^{1, q}(\Omega)\right)^{n}$ with

$$
\begin{aligned}
\operatorname{div} \mathrm{B} f & =f, \\
\|\nabla \mathrm{B} f\|_{L_{w}^{q}(\Omega)} & \leq c\|f\|_{L_{w, 0}^{q}(\Omega)} .
\end{aligned}
$$

The constant $c$ only depends on $\sigma_{1}, \sigma_{2}, q$, and $A_{q}(w)$.

If $\Omega$ is bounded, and $f \in C_{0,0}^{\infty}(\Omega)$, then $\mathrm{B} f \in\left(C_{0}^{\infty}(\Omega)\right)^{n}$.

Proof. The simple proof is based on the Decomposition Theorem 4.2, which enables us to reduce the original problem from $\Omega$ to the corresponding problem for balls. Since $\Omega$ satisfies the emanating chain condition, we can find a chain-covering $\mathscr{W}$ consisting of balls with the corresponding chains. Let $T_{i}: L_{w}^{q}(\Omega) \rightarrow L_{w, 0}^{q}\left(W_{i}\right)$ be as in Theorem 4.2 .

It has been shown in [27, Theorem 1.1, Lemma 3.3] and [15, Satz 1.25, Satz 1.42] that there exists a linear operator $B_{\text {ref }}$ which maps $C_{0,0}^{\infty}\left(B_{1}(0)\right)$ to $\left(C_{0}^{\infty}\left(B_{1}(0)\right)\right)^{n}$ and maps $L_{w, 0}^{q}\left(B_{1}(0)\right)$ to $\left(W_{w, 0}^{1, q}\left(B_{1}(0)\right)\right)^{n}$ for every $1<q<\infty$ and $w \in A_{q}$. Moreover, the operator satisfies (5.3) and (5.4) with $\Omega$ replaced by $B_{1}(0)$. By a simple translation and scaling argument it follows that there exist linear operators $\mathrm{B}_{i}: L_{w, 0}^{q}\left(W_{i}\right) \rightarrow$ $\left(W_{w, 0}^{1, q}\left(W_{i}\right)\right)^{n}$ which satisfy

$$
\begin{aligned}
\operatorname{div} \mathrm{B}_{i} g & =g, \\
\left\|\nabla \mathrm{B}_{i} g\right\|_{L_{w}^{q}\left(W_{i}\right)} & \leq c\|g\|_{L_{w, 0}^{q}\left(W_{i}\right)},
\end{aligned}
$$

for all $g \in L_{w, 0}^{q}\left(W_{i}\right)$, where $c$ only depends on $q$ and $A_{q}(w)$. Moreover, $\mathrm{B}_{i}$ maps $C_{0,0}^{\infty}\left(W_{i}\right)$ to $\left(C_{0}^{\infty}\left(W_{i}\right)\right)^{n}$.

Let $f \in L_{w}^{q}(\Omega)$. We extend $\mathrm{B}_{i} T_{i} f$ outside of $W_{i}$ by zero, so that $\mathrm{B}_{i} T_{i} f \in$ $\left(D_{w, 0}^{1, q}(\Omega)\right)^{n}$. We define our operator B by B $f:=\sum_{i \geq 0} \mathrm{~B}_{i} T_{i} f$ almost everywhere. Due to $(\mathrm{C} 3)$ and $W_{w, 0}^{1, q}\left(W_{i}\right) \hookrightarrow W_{0}^{1,1}\left(W_{i}\right)$ the sum converges in $\left(L_{\text {loc }}^{1}(\Omega)\right)^{n}$ and therefore in the sense of distributions. The same argument ensures that $\nabla \mathrm{B} f=\sum_{i} \nabla \mathrm{B}_{i} T_{i} f$ in $\left(L_{\text {loc }}^{1}(\Omega)\right)^{n \times n}$.

The estimate (5.6) and (d) of Theorem 4.2 imply

$$
\sum_{i \geq 0}\left\|\nabla \mathrm{B}_{i} T_{i} f\right\|_{L_{w}^{q}\left(W_{i}\right)}^{q} \leq c \sum_{i \geq 0}\left\|T_{i} f\right\|_{L_{w, 0}^{q}\left(W_{i}\right)}^{q} \leq c\|f\|_{L_{w, 0}^{q}(\Omega)}^{q}
$$

So with $\operatorname{supp}\left(\mathrm{B}_{i} T_{i} f\right) \subset W_{i}$ and $(\mathrm{C} 1)$, we conclude that $\mathrm{B} f=\sum_{i \geq 0} \mathrm{~B}_{i} T_{i} f$ in $\left(D_{w, 0}^{1, q}(\Omega)\right)^{n}$ and $\nabla \mathrm{B} f=\sum_{i} \nabla \mathrm{B}_{i} T_{i} f$ in $\left(L_{w}^{q}(\Omega)\right)^{n \times n}$. Moreover,

$$
\|\nabla \mathrm{B} f\|_{L_{w}^{q}(\Omega)}^{q} \leq c \sum_{i \geq 0}\left\|\nabla \mathrm{B}_{i} T_{i} f\right\|_{L_{w}^{q}\left(W_{i}\right)}^{q} \leq c\|f\|_{L_{w, 0}^{q}(\Omega)}^{q},
$$

which proves (5.4). From $\nabla \mathrm{B} f=\sum_{i} \nabla \mathrm{B}_{i} T_{i} f$ in $\left(L_{w}^{q}(\Omega)\right)^{n \times n}$ follows

$$
\operatorname{div} \mathrm{B} f=\sum_{i \geq 0} \operatorname{div} \mathrm{B}_{i} T_{i} f=\sum_{i \geq 0} T_{i} f=f
$$

for $f \in L_{w, 0}^{q}(\Omega)$. This proves (5.3). 
Assume now, that $\Omega$ is bounded and $f \in C_{0}^{\infty}(\Omega)$. Then $T_{i} f \in C_{0}^{\infty}\left(W_{i}\right)$ for all $i \geq 0$ and $T_{i} f \neq 0$ for only finitely many $i \geq 0$. Therefore, by the properties of $\mathrm{B}_{i}$, we have $\mathrm{B}_{i} T_{i} f \in\left(C_{0}^{\infty}\left(W_{i}\right)\right)^{n}$ for all $i \geq 0$ and $\mathrm{B}_{i} T_{i} f \neq 0$ for only finitely many $i \geq 0$. This proves $\mathrm{B} f \in\left(C_{0}^{\infty}(\Omega)\right)^{n}$.

Remark 5.9. The solvability of the divergence equation in bounded John domains without weights has been shown before by Acosta, Durán, and Muschietti in [1], where they construct an involved explicit solution by means of an integral operator.

For $1<q<\infty$ and $w \in A_{q}$ we define the negative, weighted homogeneous Sobolev space $D_{w}^{-1, q}(\Omega)$ to be the dual space of $D_{w^{\prime}, 0}^{1, q^{\prime}}(\Omega)$.

As a consequence of Theorem 5.2 we are able the generalize Nečas theorem on negative norms (Lions-Lemma) to the case of domains satisfying the emanating chain condition.

Theorem 5.10. (Negative norm theorem) Let $\Omega \subset \mathbf{R}^{n}$ be a domain satisfying the emanating chain condition with constants $\sigma_{1}$ and $\sigma_{2}$. Then

$$
\|\nabla f\|_{D_{w}^{-1, q}(\Omega)} \leq\|f\|_{L_{w, 0}^{q}(\Omega)} \leq c\|\nabla f\|_{D_{w}^{-1, q}(\Omega)}
$$

for all $f \in L_{w, 0}^{q}(\Omega)$. If $\Omega$ is additionally bounded, then

$$
\|f\|_{L_{w}^{q}(\Omega)} \leq c\|\nabla f\|_{D_{w}^{-1, q}(\Omega)}+\frac{c}{\operatorname{diam}(\Omega)}\|f\|_{D_{w}^{-1, q}(\Omega)}
$$

for all $f \in L_{w}^{q}(\Omega)$. The constants only depend on $\sigma_{1}, \sigma_{2}, q$, and $A_{q}(w)$.

Proof. Let $f \in L_{w, 0}^{q}(\Omega)$. Then

$$
\|\nabla f\|_{D_{w, 0}^{-1, q}(\Omega)}=\sup _{\|\mathbf{h}\|_{D_{w^{\prime}, 0}^{1, q^{\prime}}(\Omega)}^{1} \leq 1}\langle\nabla f, \mathbf{h}\rangle=\sup _{\|\mathbf{h}\|_{D_{w^{\prime}, 0}^{1, q^{\prime}}(\Omega)} \leq 1}\langle f, \operatorname{div} \mathbf{h}\rangle,
$$

which implies by Hölder's inequality

$$
\|\nabla f\|_{D_{w, 0}^{-1, q}(\Omega)} \leq\|f\|_{L_{w, 0}^{q}(\Omega)}
$$

On the other hand with (5.13) and Theorem 5.2 we get

$$
\begin{aligned}
\|\nabla f\|_{D_{w, 0}^{-1, q}(\Omega)} & \geq \sup _{\|\mathrm{B} g\|_{D_{w^{\prime}, 0}^{1, q^{\prime}}(\Omega)} \leq 1}\langle f, \operatorname{div} \mathrm{B} g\rangle=\sup _{\|\mathrm{B} g\|_{D_{w^{\prime}, 0}^{1, q^{\prime}}(\Omega)} \leq 1}\langle f, g\rangle \\
& \geq c \sup _{\|g\|_{L_{w^{\prime}, 0}^{q^{\prime}}(\Omega)} \leq 1}\langle f, g\rangle=c\|f\|_{L_{w, 0}^{q}(\Omega)},
\end{aligned}
$$

where the constant only depends on $\sigma_{1}, \sigma_{2}, q$, and $A_{q}(w)$. This proves (5.11).

Assume in the following that $\Omega$ is bounded. Let $W_{0}$ be the central cube of $\Omega$ and choose $\eta \in C_{0}^{\infty}\left(W_{0}\right)$ with $\eta \geq 0, \int_{W_{0}} \eta(x) d x=1,\|\eta\|_{\infty} \leq c /\left|W_{0}\right|$, and $\|\nabla \eta\|_{\infty} \leq$ $c /\left(\left|W_{0}\right| \operatorname{diam}\left(W_{0}\right)\right)$, where $c=c(n)$. For $f \in L_{w}^{q}(\Omega)$ we estimate with Lemma 4.25 and (5.11)

$$
\begin{aligned}
\|f\|_{L_{w}^{q}(\Omega)} & \leq c\left\|f-\langle f\rangle_{\Omega}\right\|_{L_{w, 0}^{q}(\Omega)}+|\langle f, \eta\rangle|\left\|\chi_{\Omega}\right\|_{L_{w}^{q}(\Omega)} \\
& \leq c\|\nabla f\|_{D_{w}^{-1, q}(\Omega)}+\|f\|_{D_{w}^{-1, q}(\Omega)}\|\eta\|_{D_{w^{\prime}, 0}^{1, q^{\prime}}(\Omega)}\left\|\chi_{\Omega}\right\|_{L_{w}^{q}(\Omega)} .
\end{aligned}
$$


By Remark 3.6 we have $\operatorname{diam}\left(W_{0}\right) \leq \operatorname{diam}(\Omega) \leq \sigma_{2} \operatorname{diam}\left(W_{0}\right)$. Therefore,

$$
\begin{aligned}
\|\eta\|_{D_{w^{\prime}, 0}^{1, q^{\prime}}(\Omega)}\left\|\chi_{\Omega}\right\|_{L_{w}^{q}(\Omega)} & \leq\|\nabla \eta\|_{\infty}\left\|\chi_{\Omega}\right\|_{L_{w^{\prime}}^{q^{\prime}}(\Omega)}\left\|\chi_{\Omega}\right\|_{L_{w}^{q}(\Omega)} \\
& \leq \frac{c \sigma_{2}}{\operatorname{diam}(\Omega)} \frac{\left\|\chi_{\Omega}\right\|_{L_{w^{\prime}}^{q^{\prime}}(\Omega)}\left\|\chi_{\Omega}\right\|_{L_{w}^{q}(\Omega)}}{\left|W_{0}\right|} \\
& \leq \frac{c \sigma_{2}}{\operatorname{diam}(\Omega)}\left(A_{q}(w)\right)^{\frac{1}{q}} .
\end{aligned}
$$

This and the previous estimate prove (5.12).

We now use the negative norm theorem, Theorem 5.10, to prove Korn's inequality in the first and second case: the first case is concerned with functions with zero boundary values; the second deals with the general case. In the first case we do not need any regularity conditions on our domain $\Omega$, while in the second case we need a bounded domain which satisfies the emanating chain condition. In the following, we denote by $\mathbf{D u}:=\frac{1}{2}\left((\nabla \mathbf{u})+(\nabla \mathbf{u})^{\top}\right)$ the symmetric gradient of $\mathbf{u}$, where $\mathbf{u}$ is a function from $\Omega \subset \mathbf{R}^{n}$ to $\mathbf{R}^{n}$.

Theorem 5.15. (Korn's inequality; first case) Let $\Omega \subset \mathbf{R}^{n}$ be an arbitrary domain. Let $1<q<\infty$ and $w \in A_{q}$. Then for all $\mathbf{u} \in\left(D_{w, 0}^{1, q}(\Omega)\right)^{n}$ it holds that

$$
\|\nabla \mathbf{u}\|_{L_{w}^{q}(\Omega)} \leq c\|\mathbf{D u}\|_{L_{w}^{q}(\Omega)} .
$$

The constant only depends on $q$ and $A_{q}(w)$.

Proof. Since every function $\mathbf{u} \in\left(D_{w, 0}^{1, q}(\Omega)\right)^{n}$ can be extended by zero to a function $\mathbf{u} \in\left(D_{w, 0}^{1, q}\left(\mathbf{R}^{n}\right)\right)^{n}$, it suffices to consider the case $\Omega=\mathbf{R}^{n}$.

By Theorem 5.10 and the identity $\partial_{j} \partial_{k} u_{i}=\partial_{j} D_{k i} \mathbf{u}+\partial_{k} D_{i j} \mathbf{u}-\partial_{i} D_{j k} \mathbf{u}$ (in the sense of distributions) follows

$$
\|\nabla \mathbf{u}\|_{L_{w}^{q}\left(\mathbf{R}^{n}\right)} \leq c\|\nabla \nabla \mathbf{u}\|_{D_{w}^{-1, q}\left(\mathbf{R}^{n}\right)} \leq c\|\nabla \mathbf{D u}\|_{D_{w}^{-1, q}\left(\mathbf{R}^{n}\right)} \leq c\|\mathbf{D u}\|_{L_{w}^{q}\left(\mathbf{R}^{n}\right)},
$$

where we used that $L_{w}^{p}\left(\mathbf{R}^{n}\right)=L_{w, 0}^{p}\left(\mathbf{R}^{n}\right)$.

Theorem 5.17. (Korn's inequality; second case) Let $\Omega \subset \mathbf{R}^{n}$ be a bounded domain satisfying the emanating chain condition with constants $\sigma_{1}$ and $\sigma_{2}$. Let $1<q<\infty$ and $w \in A_{q}$. Then for all $\mathbf{u} \in\left(W_{w}^{1, q}(\Omega)\right)^{n}$ it holds that

$$
\begin{gathered}
\left\|\nabla \mathbf{u}-\langle\nabla \mathbf{u}\rangle_{\Omega}\right\|_{L_{w}^{q}(\Omega)} \leq c\left\|\mathbf{D u}-\langle\mathbf{D u}\rangle_{\Omega}\right\|_{L_{w}^{q}(\Omega)}, \\
\|\nabla \mathbf{u}\|_{L_{w}^{q}(\Omega)} \leq c\left\|\mathbf{D u}-\langle\mathbf{D u}\rangle_{\Omega}\right\|_{L_{w}^{q}(\Omega)}+\frac{c}{\operatorname{diam}(\Omega)}\left\|\mathbf{u}-\langle\mathbf{u}\rangle_{\Omega}\right\|_{L_{w}^{q}(\Omega)}
\end{gathered}
$$

The constants only depend on $\sigma_{1}, \sigma_{2}, q$, and $A_{q}(w)$.

Proof. Using the identity $\partial_{j} \partial_{k} u_{i}=\partial_{j} D_{k i} \mathbf{u}+\partial_{k} D_{i j} \mathbf{u}-\partial_{i} D_{j k} \mathbf{u}$ (in the sense of distributions) the claim follows immediately from Theorem 5.10. Indeed, for bounded $\Omega$

$$
\begin{aligned}
\left\|\nabla \mathbf{u}-\langle\nabla \mathbf{u}\rangle_{\Omega}\right\|_{L_{w, 0}^{q}(\Omega)} & \leq c\|\nabla \nabla \mathbf{u}\|_{D_{w}^{-1, q}(\Omega)} \leq c\|\nabla \mathbf{D u}\|_{D_{w}^{-1, q}(\Omega)} \\
& \leq c\left\|\mathbf{D u}-\langle\mathbf{D u}\rangle_{\Omega}\right\|_{L_{w, 0}^{q}(\Omega)} .
\end{aligned}
$$

This proves (5.18). The proof of (5.19) is similar to the one of (5.12). Let $W_{0}$ be the central cube of $\Omega$ and choose $\eta \in C_{0}^{\infty}\left(W_{0}\right)$ with $\eta \geq 0, \int_{W_{0}} \eta(x) d x=1$, 
$\|\eta\|_{\infty} \leq c /\left|W_{0}\right|$, and $\|\nabla \eta\|_{\infty} \leq c /\left(\left|W_{0}\right| \operatorname{diam}\left(W_{0}\right)\right)$, where $c=c(n)$. For $\mathbf{u} \in W_{w}^{1, q}(\Omega)$ we estimate with Lemma 4.25

$$
\|\nabla \mathbf{u}\|_{L_{w}^{q}(\Omega)} \leq c\left\|\nabla \mathbf{u}-\langle\nabla \mathbf{u}\rangle_{\Omega}\right\|_{L_{w, 0}^{q}(\Omega)}+c \sum_{j, k=1}^{n}\left|\left\langle\partial_{k} u_{j}, \eta\right\rangle\right|\left\|\chi_{\Omega}\right\|_{L_{w}^{q}(\Omega)} .
$$

Since $\left\langle\partial_{k} u_{j}, \eta\right\rangle=\left\langle\partial_{k}\left(u_{j}-\left\langle u_{j}\right\rangle_{\Omega}\right), \eta\right\rangle$, we get with (5.18)

$$
\begin{aligned}
\|\nabla \mathbf{u}\|_{L_{w}^{q}(\Omega)} & \leq c\left\|\mathbf{D u}-\langle\mathbf{D u}\rangle_{\Omega}\right\|_{L_{w, 0}^{q}(\Omega)}+c \sum_{j, k=1}^{n}\left|\left\langle u_{j}-\left\langle u_{j}\right\rangle_{\Omega}, \partial_{k} \eta\right\rangle\right|\left\|\chi_{\Omega}\right\|_{L_{w}^{q}(\Omega)} \\
& \leq c\left\|\mathbf{D u}-\langle\mathbf{D u}\rangle_{\Omega}\right\|_{L_{w, 0}^{q}(\Omega)}+c\left\|\mathbf{u}-\langle\mathbf{u}\rangle_{\Omega}\right\|_{L_{w, 0}^{q}(\Omega)}\|\nabla \eta\|_{L_{w^{\prime}}^{q^{\prime}}(\Omega)}\left\|\chi_{\Omega}\right\|_{L_{w}^{q}(\Omega)} .
\end{aligned}
$$

Exactly as in (5.14) we have $\|\nabla \eta\|_{L_{w^{\prime}}^{q^{\prime}}(\Omega)}\left\|\chi_{\Omega}\right\|_{L_{w}^{q}(\Omega)} \leq c / \operatorname{diam}(\Omega)$ where $c$ depends only on $\sigma_{2}, q$, and $A_{q}(w)$. This and the previous estimate prove (5.19).

Remark 5.20. Korn's inequality in the second case in the form of (5.19) for John domains without weights has been shown before by Acosta, Durán, and Muschietti in [1].

5.3. Local Fefferman-Stein inequality. Let $\Omega \subset \mathbf{R}^{n}$ be a domain satisfying the emanating chain condition. For $f \in L^{1}(\Omega), \sigma \geq 1$ or $f \in L_{\text {loc }}^{1}(\Omega), \sigma>1$ we define the restricted maximal function $M_{\mathrm{res}, \Omega, \sigma} f: \mathbf{R}^{n} \rightarrow[0, \infty]$ and the restricted sharp maximal function $M_{\mathrm{res}, \Omega, \sigma}^{\sharp} f: \mathbf{R}^{n} \rightarrow[0, \infty]$ by

$$
\begin{aligned}
& \left(M_{\mathrm{res}, \Omega, \sigma} f\right)(x):=\sup _{Q \ni x: \sigma Q \subset \Omega} f_{Q}|f(y)| d y, \\
& \left(M_{\mathrm{res}, \Omega, \sigma}^{\sharp} f\right)(x):=\sup _{Q \ni x: \sigma Q \subset \Omega} f_{Q}\left|f(y)-\langle f\rangle_{Q}\right| d y,
\end{aligned}
$$

for all $x \in \Omega$, where the supremum is taken over all cubes $Q \subset \mathbf{R}^{n}$ with $\sigma Q \subset \Omega$ and $x \in Q$. We extend $M_{\mathrm{res}, \Omega, \sigma} f$ and $M_{\mathrm{res}, \Omega, \sigma}^{\sharp} f$ outside of $\Omega$ by zero.

Iwaniec [19, Lemma 4] proved the following local version of the Fefferman and Stein inequality.

Lemma 5.21. Let $Q_{0} \subset \mathbf{R}^{n}$ be a cube, $1 \leq q<\infty$, and $f \in L^{1}\left(Q_{0}\right)$. If $M_{\text {res, } Q_{0}, 1}^{\sharp} f \in L^{q}\left(Q_{0}\right)$, then $M_{\text {res, } Q_{0}, 1} f \in L^{q}\left(Q_{0}\right)$ and

$$
\left(f_{Q_{0}}\left|M_{\mathrm{res}, Q_{0}, 1} f\right|^{q} d x\right)^{\frac{1}{q}} \leq 10^{5 n q}\left(f_{Q_{0}}\left|M_{\mathrm{res}, Q_{0}, 1}^{\sharp} f\right|^{q} d x\right)^{\frac{1}{q}}+10^{n+1} f_{Q_{0}}|f| d x .
$$

This result has a nice consequence for the space $L_{0}^{q}\left(Q_{0}\right)$ of functions with mean value zero.

Corollary 5.22. Let $Q_{0} \subset \mathbf{R}^{n}$ be a cube, $1 \leq q<\infty$, and let $f \in L_{0}^{1}\left(Q_{0}\right)$. If $M_{\text {res, } Q_{0}, 1}^{\sharp} f \in L^{q}\left(Q_{0}\right)$, then $f \in L_{0}^{q}\left(Q_{0}\right)$ and

$$
\|f\|_{L_{0}^{q}\left(Q_{0}\right)} \leq 10^{5 n q+n+1}\left\|M_{\text {res }, Q_{0}, 1}^{\sharp} f\right\|_{L^{q}\left(Q_{0}\right)} .
$$

Proof. Since $\langle f\rangle_{Q_{0}}=0$, we have $f_{Q_{0}}|f| d y \leq M_{\mathrm{res}, Q_{0}, 1}^{\sharp} f(x)$ for all $x \in Q_{0}$. Taking the mean value of this inequality we get also using $f_{Q_{0}}\left|M_{\text {res, } Q_{0}, 1}^{\sharp} f\right| d x \leq$ 
$\left(f_{Q_{0}}\left|M_{\mathrm{res}, Q_{0}, 1}^{\sharp} f\right|^{q} d x\right)^{\frac{1}{q}}$ and Lemma 5.21

$$
\left(f_{Q_{0}}\left|M_{\mathrm{res}, Q_{0}, 1} f\right|^{q} d x\right)^{\frac{1}{q}} \leq 10^{5 n q+n+1}\left(f_{Q_{0}}\left|M_{\mathrm{res}, Q_{0}, 1}^{\sharp} f\right|^{q} d x\right)^{\frac{1}{q}} .
$$

Multiplying by $\left|Q_{0}\right|^{\frac{1}{q}}$ and using $|f| \leq M_{\text {res }, Q_{0}, 1} f$ proves the claim.

We generalize this result to domains satisfying the emanating chain condition including the weighted case.

Theorem 5.23. Let $\Omega \subset \mathbf{R}^{n}$ be a bounded domain satisfying the emanating chain condition with constants $\sigma_{1}$ and $\sigma_{2}$. Let $1<q<\infty, w \in A_{q}, f \in L_{\mathrm{loc}}^{1}(\Omega)$, and $\sigma_{1}>1$. If $M_{\text {res, }, \Omega \sigma_{1}}^{\sharp} f \in L_{w}^{q}(\Omega)$, then $f \in L_{w}^{q}(\Omega)$ and

$$
\left\|f-\langle f\rangle_{\Omega}\right\|_{L_{w, 0}^{q}(\Omega)} \leq c\left\|M_{\mathrm{res}, \Omega, \sigma_{1}}^{\sharp} f\right\|_{L_{w}^{q}(\Omega)} .
$$

The constant $c$ depends only on $\sigma_{1}, \sigma_{2}, q$, and $A_{q}(w)$. If $f \in L^{1}(\Omega)$, then it suffices to assume $\sigma_{1} \geq 1$.

Using our Decomposition Theorem 4.2 and Corollary 5.22 we would be able to prove the unweighted version of Theorem 5.23. However, for the proof of the weighted version we need the following weighted version of Corollary 5.22.

Lemma 5.25. Let $Q_{0} \subset \mathbf{R}^{n}$ be a cube, $1 \leq q<\infty, w \in A_{q}$, and $f \in L_{0}^{1}\left(Q_{0}\right)$. If $M_{\text {res }, Q_{0}, 1}^{\sharp} f \in L_{w}^{q}\left(Q_{0}\right)$, then $f \in L_{w, 0}^{q}\left(Q_{0}\right)$ and

$$
\|f\|_{L_{w, 0}^{q}\left(Q_{0}\right)} \leq c\left\|M_{r e s, Q_{0}, 1}^{\sharp} f\right\|_{L_{w}^{q}\left(Q_{0}\right)},
$$

where the constant only depends on $q$ and $A_{q}(w)$.

Since we want to show in this section how our Decomposition Theorem 4.2 and Lemma 5.25 imply Theorem 5.23, we postpone the proof of Lemma 5.25 to the appendix and continue with the proof of Theorem 5.23.

Proof of Theorem 5.23. Let $1<q<\infty, w \in A_{q}$, and $f \in L_{\mathrm{loc}}^{1}(\Omega)$ with $M_{\mathrm{res}, \Omega, \sigma_{1}}^{\sharp} f \in L_{w}^{q}(\Omega)$. Since $w \in A_{q}$, we have $w^{\prime}=w^{\frac{1}{1-q}} \in A_{q^{\prime}}$.

Let $h \in C_{0,0}^{\infty}(\Omega)$, then by Theorem 4.2 we can decompose $h$ in $L_{w^{\prime}, 0}^{q^{\prime}}(\Omega)$ into sum of functions $T_{i} h \in C_{0,0}^{\infty}\left(W_{i}\right)$ such that

$$
\left(\sum_{i \geq 0}\left\|T_{i} h\right\|_{L_{w^{\prime}, 0}^{q^{\prime}\left(W_{i}\right)}}^{q^{\prime}}\right)^{\frac{1}{q^{\prime}}} \leq c\|h\|_{L_{w^{\prime}, 0}^{q^{\prime}}(\Omega)} .
$$

Moreover, Theorem 4.2 ensures (using the boundedness of $\Omega$ ) that only finitely many summands of $h=\sum_{i \geq 0} T_{i} h$ are non-zero.

Since $f \in L_{\text {loc }}^{1}(\Omega)$ and $h \in C_{0,0}^{\infty}(\Omega),\langle f, h\rangle$ is well defined. Only finitely many of the $T_{i} h$ are non-zero and $\int_{W_{i}} T_{i} h d x=0$, so we have

$$
\langle f, h\rangle=\sum_{i}\left\langle f, T_{i} h\right\rangle=\sum_{i}\left\langle f-\langle f\rangle_{W_{i}}, T_{i} h\right\rangle,
$$


where we also used that $f \in L^{1}\left(W_{i}\right)$, since $\sigma_{1}>1$ and consequently $\overline{W_{1}} \subset \sigma_{1} W_{i} \subset \Omega$. We estimate with Lemma 5.25 (in the second step)

$$
\begin{aligned}
\langle f, h\rangle & \leq \sum_{i}\left\|f-\langle f\rangle_{W_{i}}\right\|_{L_{w, 0}^{q}\left(W_{i}\right)}\left\|T_{i} h\right\|_{L_{w^{\prime}, 0}^{q^{\prime}}\left(W_{i}\right)} \\
& \leq c \sum_{i}\left\|M_{\mathrm{res}, W_{i}, 1}^{\sharp} f\right\|_{L_{w}^{q}\left(W_{i}\right)}\left\|T_{i} h\right\|_{L_{w^{\prime}, 0}^{q^{\prime}}\left(W_{i}\right)} \\
& \leq c \sum_{i}\left\|\chi_{W_{i}} M_{\mathrm{res}, \Omega, \sigma_{1}}^{\sharp} f\right\|_{L_{w}^{q}(\Omega)}\left\|T_{i} h\right\|_{L_{w^{\prime}, 0}^{q^{\prime}}\left(W_{i}\right)} \\
& \leq c\left(\sum_{i}\left\|\chi_{W_{i}} M_{\mathrm{res}, \Omega, \sigma_{1}}^{\sharp} f\right\|_{L_{w}^{q}(\Omega)}^{q}\right)^{\frac{1}{q}}\left(\sum_{i}\left\|T_{i} h\right\|_{L_{w^{\prime}, 0}^{q^{\prime}}\left(W_{i}\right)}^{q^{\prime}}\right)^{\frac{1}{q^{\prime}}} \\
& \leq c\left\|M_{\mathrm{res}, \Omega, \sigma_{1}}^{\sharp} f\right\|_{L_{w}^{q}(\Omega)}\|h\|_{L_{w^{\prime}, 0}^{q^{\prime}}(\Omega)}
\end{aligned}
$$

for all $h \in C_{0,0}^{\infty}(\Omega)$, where we have used in the last step $\sum_{W \in \mathscr{W}} \chi_{\sigma_{1} W} \leq \sigma_{2} \chi_{\Omega}$ and (5.26).

We have shown that the mapping $T: h \mapsto\langle f, h\rangle$ is bounded as a mapping from $\left(C_{0,0}^{\infty}(\Omega),\|\cdot\|_{L_{w^{\prime}, 0}^{q^{\prime}}(\Omega)}\right)$ to $\mathbf{R}$. Since $C_{0,0}^{\infty}(\Omega)$ is dense in $L_{w^{\prime}, 0}^{q^{\prime}}(\Omega)$, there exists a unique $\bar{T} \in\left(L_{w^{\prime}, 0}^{q^{\prime}}(\Omega)\right)^{*}$ with $\bar{T}(f)=\langle f, h\rangle$ for all $h \in C_{0,0}^{\infty}(\Omega)$. Since $\left(L_{w^{\prime}, 0}^{q^{\prime}}(\Omega)\right)^{*} \cong L_{w, 0}^{q}(\Omega)$ there exists $\bar{f} \in L_{w, 0}^{q}(\Omega)$ such that $T(h)=\langle\bar{f}, h\rangle$ for all $h \in L_{w^{\prime}, 0}^{q^{\prime}}(\Omega)$. In particular, we have $\langle\bar{f}-f, h\rangle=0$ for all $h \in C_{0,0}^{\infty}(\Omega)$. Since the operator div maps $C_{0}^{\infty}\left(\Omega ; \mathbf{R}^{n}\right)$ into $C_{0,0}^{\infty}(\Omega)$, if follows that $\langle\bar{f}-f$, $\operatorname{div} \mathbf{w}\rangle=0$ for all $\mathbf{w} \in C_{0}^{\infty}\left(\Omega, \mathbf{R}^{n}\right)$. This implies that $\nabla(\bar{f}-f)=0$ in the sense of distributions. So by the Theorem of DuBoisReymond there exists a constant $c_{0} \in \mathbf{R}$ such that $f=\bar{f}+c_{0}$. Thus, $f \in L_{w}^{q}(\Omega)$ and $\langle f\rangle_{\Omega}=c_{0}$. From $\left\langle f-\langle f\rangle_{\Omega}, h\right\rangle=\langle\bar{f}, h\rangle=\langle f, h\rangle$ for all $h \in C_{0,0}^{\infty}(\Omega)$ and (5.27) follows (5.24).

Remark 5.28. Note that Theorem 5.23 generalizes Corollary 5.22 by Iwaniec even in the unweighted case in the sense that $M_{\mathrm{res}, \Omega, 1}^{\sharp}$ is replaced by $M_{\mathrm{res}, \Omega, \sigma_{1}}^{\sharp}$. So the supremum is only taken over cubes which are after enlargement by $\sigma_{1}>1$ still in $\Omega$. This is in particular of interest in applications to partial differential equations.

Remark 5.29. It is possible to deduce Theorem 5.23 from [20, Theorem 3] together with Lemma 5.25. However, our motivation was to show that our Decomposition Theorem 4.2 also provides a simple proof of the result.

Remark 5.30. It is also possible to use Theorem 5.23 for another proof of Korn's inequality Theorem 5.17. We refer to [10, section 5], where Korn's inequality is deduced from Theorem 5.23 for the Sobolev spaces of variable exponents $W^{1, p(\cdot)}(\Omega)$. The arguments for $D_{w, 0}^{1, q}(\Omega)$ are exactly the same.

\section{Extrapolation}

Using the extrapolation technique of Rubio de Francia [26] it is possible to extend our result to many other Banach function spaces. We do this in the following for unweighted Orlicz spaces, since we need these results in a forthcoming article for the numerical analysis of the $q$-Stokes system. However, the technique can be applied 
to many other situations. We refer to [7] for a detailed study of the extrapolation technique.

The following definitions and results are standard in the context of Orlicz spaces [22]. A real function $\varphi: \mathbf{R}^{\geq 0} \rightarrow \mathbf{R}^{\geq 0}$ is said to be an $\mathrm{N}$-function if it satisfies the following conditions: There exists the derivative $\varphi^{\prime}$ of $\varphi$. This derivative is right continuous, non-decreasing and satisfies $\varphi^{\prime}(0)=0$ and $\varphi^{\prime}(t)>0$ for $t>0$. Especially, $\varphi$ is convex.

We say that $\varphi$ satisfies the $\Delta_{2}$-condition, if there exists $c_{1}>0$ such that for all $t \geq 0$ holds $\varphi(2 t) \leq c_{1} \varphi(t)$. By $\Delta_{2}(\varphi)$ we denote the smallest constant $c_{1}$. Since $\varphi(t) \leq \varphi(2 t)$, the $\Delta_{2}$ condition is equivalent to $\varphi(2 t) \sim \varphi(t)$. For a family $\varphi_{\lambda}$ of N-functions we define $\Delta_{2}\left(\left\{\varphi_{\lambda}\right\}\right):=\sup _{\lambda} \Delta_{2}\left(\varphi_{\lambda}\right)$. By $\left(\varphi^{\prime}\right)^{-1}: \mathbf{R}^{\geq 0} \rightarrow \mathbf{R}^{\geq 0}$ we denote the function

$$
\left(\varphi^{\prime}\right)^{-1}(t):=\sup \left\{u \in \mathbf{R}^{\geq 0}: \varphi^{\prime}(u) \leq t\right\} .
$$

If $\varphi^{\prime}$ is strictly increasing then $\left(\varphi^{\prime}\right)^{-1}$ is the inverse function of $\varphi^{\prime}$. Then $\varphi^{*}: \mathbf{R}^{\geq 0} \rightarrow$ $\mathbf{R}^{\geq 0}$ with

$$
\varphi^{*}(t):=\int_{0}^{t}\left(\varphi^{\prime}\right)^{-1}(s) d s
$$

is again an $\mathrm{N}$-function and $\left(\varphi^{*}\right)^{\prime}(t)=\left(\varphi^{\prime}\right)^{-1}(t)$ for $t>0$. It is the complementary function of $\varphi$. Note that $\left(\varphi^{*}\right)^{*}=\varphi$.

For an open set $\Omega \subset \mathbf{R}^{n}$ and an $\mathrm{N}$-function $\varphi$ with $\Delta_{2}(\varphi)<\infty$ we define

$$
L^{\varphi}(\Omega):=\left\{f \in L_{\mathrm{loc}}^{1}(\Omega): \int_{\mathbf{R}^{n}} \varphi(|f(x)|) d x<\infty\right\} .
$$

Note that $L^{\varphi}(\Omega)$ becomes a Banach space with the norm

$$
\|f\|_{L^{\varphi}(\Omega)}:=\inf \left\{\lambda>0: \int_{\mathbf{R}^{n}} \varphi\left(\frac{|f(x)|}{\lambda}\right) d x \leq 1\right\} .
$$

For $\Omega$ bounded we define $L_{0}^{\varphi}(\Omega):=\left\{f \in L^{\varphi}(\Omega): \int_{\Omega} f(x) d x=0\right\}$ and for $\Omega$ unbounded we define $L_{0}^{\varphi}(\Omega):=L^{\varphi}(\Omega)$. For this we have used that $L^{\varphi}(\Omega) \hookrightarrow L^{1}(\Omega)$ for bounded domains. In both cases $L_{0}^{\varphi}(\Omega)$ is the closure of all $L^{\varphi}(\Omega)$-functions with compact support in $\Omega$ and vanishing integral. We define $W^{1, \varphi}(\Omega)$ to be the space of functions $f \in L^{\varphi}(\Omega)$ with $\nabla f \in L^{\varphi}(\Omega)$. The space is equipped with the norm $\|f\|_{W^{1, \varphi}(\Omega)}:=\|f\|_{L^{\varphi}(\Omega)}+\|\nabla f\|_{L^{\varphi}(\Omega)}$. By $W_{0}^{1, \varphi}(\Omega)$ we denote the completion of $C_{0}^{\infty}(\Omega)$ within $W^{1, \varphi}(\Omega)$. For a domain $\Omega \subset \mathbf{R}^{n}$ we define $D_{0}^{1, \varphi}(\Omega)$ to be the closure of $C_{0}^{\infty}(\Omega)$ with respect to the gradient norm $\|\nabla \cdot\|_{L^{\varphi}(\Omega)}$. Note that if $\Omega$ is bounded, then $W_{0}^{1, \varphi}(\Omega)=D_{0}^{1, \varphi}(\Omega)$.

We need the following extrapolation result of Cruz-Uribe, Martell, and Pérez $[8$, Theorem 3.1].

Proposition 6.1. Let $1<q<\infty$ and let $\mathscr{F}$ be a family of couples $(f, g)$ from $L_{\text {loc }}^{1}\left(\mathbf{R}^{n}\right) \times L_{\text {loc }}^{1}\left(\mathbf{R}^{n}\right)$ such that there exists an $A_{q}$-consistent constant $K_{1}>0$ such that

$$
\int_{\mathbf{R}^{n}}|f(x)|^{q} w(x) d x \leq K_{1} \int_{\mathbf{R}^{n}}|g(x)|^{q} w(x) d x
$$


for all $(f, g) \in \mathscr{F}$ and all $w \in A_{q}$. Then for all $N$-functions $\varphi$ with $\Delta_{2}(\varphi), \Delta_{2}\left(\varphi^{*}\right)<$ $\infty$, there exists $K_{2}>0$ such that for all $(f, g) \in \mathscr{F}$ holds

$$
\begin{gathered}
\|f\|_{L^{\varphi}\left(\mathbf{R}^{n}\right)} \leq K_{2} K_{1}\|g\|_{L^{\varphi}\left(\mathbf{R}^{n}\right)}, \\
\int_{\mathbf{R}^{n}} \varphi(|f|) d x \leq K_{2} \int_{\mathbf{R}^{n}} \varphi\left(K_{1}|g|\right) d x .
\end{gathered}
$$

The constant $K_{2}$ only depends on $\Delta_{2}(\varphi), \Delta_{2}\left(\varphi^{*}\right)$, and $q$.

Proof. There are two details which differ from the original version of the proposition in [8, Theorem 3.1], which we explain in the following.

First, the linear dependence in (6.3) of the constant $K_{1}$ is omitted. However, the dependence follows if we apply [8, Theorem 3.1] to the family $\mathscr{F}_{2}:=$ $\left\{\left(f, K_{1} g\right):(f, g) \in \mathscr{F}\right\}$, which satisfies (6.2) with $K_{1}$ replaced by 1 .

Second, in the original version it is required for (6.3) that the left-hand sides of (6.3) are already finite. This condition, however, is not needed. For $k \in \mathbf{N}$ we define the family $\mathscr{F}_{3}:=\left\{\left(f_{k}, g\right):(f, g) \in \mathscr{F}, k \in \mathbf{N}\right\}$, where $f_{k}:=\chi_{B_{k}(0) \cap\{|f| \leq k\}} f$. Then the family $\mathscr{F}_{3}$ satisfies (6.2). Since the left-hand side of (6.3) is finite for all $\left(f_{k}, g\right) \in \mathscr{F}_{3}$, we get that

$$
\begin{aligned}
\left\|f_{k}\right\|_{L^{\varphi}\left(\mathbf{R}^{n}\right)} & \leq K_{2} K_{1}\|g\|_{L^{\varphi}\left(\mathbf{R}^{n}\right)}, \\
\int_{\mathbf{R}^{n}} \varphi\left(\left|f_{k}\right|\right) d x & \leq K_{2} \int_{\mathbf{R}^{n}} \varphi\left(K_{1}|g|\right) d x .
\end{aligned}
$$

We will derive now from this (6.3). If $g \notin L^{\varphi}\left(\mathbf{R}^{n}\right)$, then the right-hand sides of (6.3) are both $\infty$ and there is nothing to show. So we can assume in the following that $g \in$ $L^{\varphi}\left(\mathbf{R}^{n}\right)$, which implies that the right-hand sides of (6.3) are both finite. Moreover, $0 \leq\left|f_{k}\right| \leq|f|$ and $\left|f_{k}\right| \nearrow|f|$ pointwise, so we get by the Fatou property of $L^{\varphi}\left(\mathbf{R}^{n}\right)$ (see [22]) for the norm and the modular that $\left\|f_{k}\right\|_{\varphi} \rightarrow\|f\|_{\varphi}$ and $\int_{\mathbf{R}^{n}} \varphi\left(\left|f_{k}\right|\right) d x \rightarrow$ $\int_{\mathbf{R}^{n}} \varphi(|f|) d x$, respectively. In particular, this and (6.4) imply (6.3).

Using Proposition 6.1 and our result for the weighted Lebesgue spaces $L_{w}^{q}(\Omega)$, we immediately get the corresponding result for unweighted Orlicz spaces. Let us exemplary present Theorem 5.1, Theorem 5.2, Theorem 5.15 and Theorem 5.17 for unweighted Orlicz spaces. For related results see [17].

Theorem 6.5. Let $\Omega \subset \mathbf{R}^{n}$ be a bounded domain satisfying the emanating chain condition with constants $\sigma_{1}$ and $\sigma_{2}$. Let $\varphi$ be an N-function with $\Delta_{2}(\varphi), \Delta_{2}\left(\varphi^{*}\right)<\infty$. Then for all $f \in W^{1, \varphi}(\Omega)$ holds

$$
\begin{gathered}
\int_{\Omega} \varphi\left(\left|f-\langle f\rangle_{\Omega}\right|\right) d x \leq c \int_{\Omega} \varphi(\operatorname{diam}(\Omega)|\nabla f|) d x \\
\left\|f-\langle f\rangle_{\Omega}\right\|_{L^{\varphi}(\Omega)} \leq c \operatorname{diam}(\Omega)\|\nabla f\|_{L^{\varphi}(\Omega)} .
\end{gathered}
$$

The constants only depends on $\sigma_{1}, \sigma_{2}, \Delta_{2}(\varphi)$, and $\Delta_{2}\left(\varphi^{*}\right)$.

Theorem 6.6. Let $\Omega \subset \mathbf{R}^{n}$ be a domain satisfying the emanating chain condition with constants $\sigma_{1}$ and $\sigma_{2}$. Then there exists a linear operator B: $C_{0}^{\infty}(\Omega) \rightarrow$ $L_{\text {loc }}^{1}(\Omega)$ which extends uniquely for all $N$-functions $\varphi$ with $\Delta_{2}(\varphi), \Delta_{2}\left(\varphi^{*}\right)<\infty$ to an 
operator B : $L_{0}^{\varphi}(\Omega) \rightarrow\left(D_{0}^{1, \varphi}(\Omega)\right)^{n}$ with

$$
\begin{aligned}
\operatorname{div} \mathrm{B} f & =f \\
\|\nabla \mathrm{B} f\|_{L^{\varphi}(\Omega)} & \leq c\|f\|_{L_{0}^{\varphi}(\Omega)}, \\
\int_{\Omega} \varphi(|\nabla \mathrm{B} f|) d x & \leq c \int_{\Omega} \varphi(|f|) d x .
\end{aligned}
$$

The constant $c$ only depends on $\sigma_{1}, \sigma_{2}, \Delta_{2}(\varphi)$, and $\Delta_{2}\left(\varphi^{*}\right)$.

If $\Omega$ is bounded, and $f \in C_{0,0}^{\infty}(\Omega)$ with then $\mathrm{B} f \in\left(C_{0}^{\infty}(\Omega)\right)^{n}$.

Theorem 6.10. (Korn's inequality; first case) Let $\Omega \subset \mathbf{R}^{n}$ be an arbitrary domain. Let $\varphi$ be an $N$-function with $\Delta_{2}(\varphi), \Delta_{2}\left(\varphi^{*}\right)<\infty$. Then for all $\mathbf{u} \in\left(D_{0}^{1, \varphi}(\Omega)\right)^{n}$ it holds that

$$
\begin{gathered}
\|\nabla \mathbf{u}\|_{L^{\varphi}(\Omega)} \leq c\|\mathbf{D u}\|_{L^{\varphi}(\Omega)}, \\
\int_{\Omega} \varphi(|\nabla \mathbf{u}|) d x \leq c \int_{\Omega} \varphi(|\mathbf{D u}|) d x .
\end{gathered}
$$

The constants only depend on $\sigma_{1}, \sigma_{2}, \Delta_{2}(\varphi)$, and $\Delta_{2}\left(\varphi^{*}\right)$.

Theorem 6.13. (Korn's inequality; second case) Let $\Omega \subset \mathbf{R}^{n}$ be a bounded domain satisfying the emanating chain condition with constants $\sigma_{1}$ and $\sigma_{2}$. Let $\varphi$ be an $N$-function with $\Delta_{2}(\varphi), \Delta_{2}\left(\varphi^{*}\right)<\infty$. Then for all $\mathbf{u} \in\left(W^{1, \varphi}(\Omega)\right)^{n}$ it holds that

$$
\begin{gathered}
\left\|\nabla \mathbf{u}-\langle\nabla \mathbf{u}\rangle_{\Omega}\right\|_{L^{\varphi}(\Omega)} \leq c\left\|\mathbf{D u}-\langle\mathbf{D u}\rangle_{\Omega}\right\|_{L^{\varphi}(\Omega)}, \\
\int_{\Omega} \varphi\left(\left|\nabla \mathbf{u}-\langle\nabla \mathbf{u}\rangle_{\Omega}\right|\right) d x \leq c \int_{\Omega} \varphi\left(\left|\mathbf{D u}-\langle\mathbf{D u}\rangle_{\Omega}\right|\right) d x .
\end{gathered}
$$

The constants only depend on $\sigma_{1}, \sigma_{2}, \Delta_{2}(\varphi)$, and $\Delta_{2}\left(\varphi^{*}\right)$.

Remark 6.16. Also our Decomposition Theorem 4.2 holds in the context of Orlicz spaces. We just need to replace $L_{w, 0}^{q}(\Omega)$ by $L_{0}^{\varphi}(\Omega)$ and (4.5) by

$$
\frac{1}{c} \int \varphi(|f|) d x \leq \sum_{i \geq 0} \int_{W_{i}} \varphi\left(\left|T_{i} f\right|\right) d x \leq c \int \varphi(|f|) d x .
$$

\section{Appendix (proof of Lemma 5.25)}

In the following we present the proof of Lemma 5.25. It is based on the ideas of [19, Lemma 4]. We need the following version of the Calderón-Zygmund decomposition by Iwaniec which is contained in the proof of [19, Lemma 3].

Lemma 7.1. Let $Q_{0} \subset \mathbf{R}^{n}$ be a cube and let $f \in L^{1}\left(Q_{0}\right)$. For every $\alpha \geq\langle|f|\rangle_{Q_{0}}$ there exist disjoint cubes $Q_{j}^{\alpha} \subset Q_{0}, j \in \mathbf{N}$ such that

(a) $\alpha<f_{Q_{j}^{\alpha}}|f| d x \leq 2^{n} \alpha$

(b) If $\alpha \geq \beta \geq\langle|f|\rangle_{Q_{0}}$, then each cube $Q_{j}^{\alpha}$ is a subset of a cube from the family $\left\{Q_{j}^{\beta}: j \in \mathbf{N}\right\}$

(c) $|f| \leq \alpha$ on the set $Q_{0} \backslash \bigcup_{j} Q_{j}^{\alpha}$,

(d) $\bigcup_{j} Q_{j}^{\alpha} \subset\left\{M_{\text {res }, Q_{0}, 1} f>\alpha\right\}$ for all $\alpha \geq\langle|f|\rangle_{Q_{0}}$,

(e) $\left\{M_{\text {res }, Q_{0}, 1} f>5^{n} \alpha\right\} \subset \bigcup_{j} 5 Q_{j}^{\alpha}$ for all $\alpha \geq\langle|f|\rangle_{Q_{0}}$.

We are now prepared to generalize Lemma 5.21 to the weighted case. The proof is similar to the unweighted case by Iwaniec. 
Lemma 7.2. Let $Q_{0} \subset \mathbf{R}^{n}$ be a cube, $1 \leq q<\infty, w \in A_{\infty}$, and let $f \in L^{1}\left(Q_{0}\right)$. If $M_{\mathrm{res}, Q_{0}, 1}^{\sharp} f \in L_{w}^{q}\left(Q_{0}\right)$, then $M_{\mathrm{res}, Q_{0}, 1} f \in L_{w}^{q}\left(Q_{0}\right)$ and

$$
\int_{Q_{0}}\left|M_{\mathrm{res}, Q_{0}, 1} f\right|^{q} w d x \leq c \int_{Q_{0}}\left|M_{\mathrm{res}, Q_{0}, 1}^{\sharp} f\right|^{q} w d x+c w\left(Q_{0}\right)\left(f_{Q_{0}}|f| d x\right)^{q} .
$$

The constant $c$ depends only on $n$ and the $A_{\infty}$-constant of $w$.

Proof. Since $w \in A_{\infty}$, the exists a constant $c_{0}$, which only depends on the $A_{\infty}$ constant of $w$ such that $\omega(5 Q) \leq c_{0} \omega(Q)$ for any cube $Q \subset \mathbf{R}^{n}$, see [31, V 1.6, p. 196]. We shall show first the following redistributional estimate: For every $\varepsilon>0$ there exists $\delta>0$ such that

$$
\begin{aligned}
w\left(\left\{M_{\mathrm{res}, Q_{0}, 1} f>5^{n} \alpha\right\}\right) \leq & c_{0} w\left(\left\{M_{\mathrm{res}, Q_{0}, 1}^{\sharp} f(x)>\delta \alpha\right\}\right) \\
& +c_{0} \varepsilon w\left(\left\{M_{\mathrm{res}, Q_{0}, 1} f>2^{-n-1} \alpha\right\}\right)
\end{aligned}
$$

for all $f \in L^{1}\left(Q_{0}\right)$ and all $\alpha \geq 2^{n+1}\langle|f|\rangle_{Q_{0}}$. The constant $\delta$ only depends on $\varepsilon, n$, and the $A_{\infty}$-constant of $w$. Using 7.1 of Lemma 7.1 and $\omega(5 Q) \leq c_{0} \omega(Q)$ it suffices to show

$$
\sum_{j} w\left(Q_{j}^{\alpha}\right) \leq w\left(\left\{M_{\mathrm{res}, Q_{0}, 1}^{\sharp} f(x)>\delta \alpha\right\}\right)+\varepsilon w\left(\left\{M_{\mathrm{res}, Q_{0}, 1} f>2^{-n-1} \alpha\right\}\right)
$$

Fix $Q \in\left\{Q_{j}^{\alpha 2^{-n-1}}: j \in \mathbf{N}\right\}$. Since $w \in A_{\infty}$, there exists an $\varepsilon_{2}>0$ such that if $E \subset Q$ with $|E| \leq \varepsilon_{2}|Q|$, then $w(E) \leq \varepsilon w(Q)$, see [31, V 1.7, p. 196]. Note that $\varepsilon_{2}$ only depends on $\varepsilon$ and the $A_{\infty}$-constant of $w$ but is independent of $Q$ and $E$.

By 7.1 of Lemma 7.1 follows $\left|\langle f\rangle_{Q}\right| \leq \frac{\alpha}{2}$ and $f_{Q_{j}^{\alpha}}|f| d x \geq \alpha$ for every $j \in \mathbf{N}$. This implies

$$
f_{Q_{j}^{\alpha}}\left|f-\langle f\rangle_{Q}\right| d x \geq f_{Q_{j}^{\alpha}}|f| d x-\left|\langle f\rangle_{Q}\right| \geq \frac{\alpha}{2}
$$

for all $j \in \mathbf{N}$. Multiplying by $\left|Q_{j}^{\alpha}\right|$ and summing over all $Q_{j}^{\alpha}$ which are contained in $Q$ we get

$$
\sum_{Q_{j}^{\alpha} \subset Q}\left|Q_{j}^{\alpha}\right| \leq \frac{2}{\alpha} \int_{Q}\left|f-\langle f\rangle_{Q}\right| d x
$$

Now, we consider two cases:

Case $f_{Q}\left|f-\langle f\rangle_{Q}\right| d x \leq \frac{\alpha}{2} \varepsilon_{2}$ : Then $\sum_{Q_{j}^{\alpha} \subset Q}\left|Q_{j}^{\alpha}\right| \leq \varepsilon_{2}|Q|$. By choice of $\varepsilon_{2}$ follows $\sum_{Q_{j}^{\alpha} \subset Q} w\left(Q_{j}^{\alpha}\right) \leq \varepsilon w(Q)$, where we have used that the $Q_{j}^{\alpha}, j \in \mathbf{N}$ are disjoint.

Case $f_{Q}\left|f-\langle f\rangle_{Q}\right| d x>\frac{\alpha}{2} \varepsilon_{2}$ : This means $M_{\mathrm{res}, Q_{0}, 1}^{\sharp} f(x)>\frac{\alpha}{2} \varepsilon_{2}=\delta \alpha$ for all points $x \in Q$, where $\delta:=\frac{1}{2} \varepsilon_{2}$. In particular, $Q \subset\left\{M_{\mathrm{res}, Q_{0}, 1}^{\sharp} f>\delta \alpha\right\}$ and

$$
\sum_{Q_{j}^{\alpha} \subset Q} w\left(Q_{j}^{\alpha}\right) \leq w(Q)=w\left(Q \cap\left\{M_{\mathrm{res}, Q_{0}, 1}^{\sharp} f>\delta \alpha\right\}\right) .
$$

Combining these results yields

$$
\sum_{Q_{j}^{\alpha} \subset Q} w\left(Q_{j}^{\alpha}\right) \leq w\left(Q \cap\left\{M_{\mathrm{res}, Q_{0}, 1}^{\sharp} f>\delta \alpha\right\}\right)+\varepsilon w(Q) .
$$


Appealing to property 7.1 in Lemma 7.1 and $\alpha \geq 2^{n+1}\langle|f|\rangle_{Q_{0}}$ we see that, after summing the last estimate over the pairwise disjoint cubes $Q \in\left\{Q_{j}^{\alpha 2^{-n-1}}: j \in \mathbf{N}\right\}$, all cubes from $\left\{Q_{j}^{\alpha}: j \in \mathbf{N}\right\}$ are counted exactly once. In particular, we obtain (7.4) and therefore (7.3).

The rest of the proof is standard and is word by word the same as the one for the unweighted case of Iwaniec [19, Lemma 4]. The idea is to integrate (7.3) over $\alpha$ from $2^{n+1}\langle|f|\rangle_{Q_{0}}$ to $\infty$ and use

$$
\int_{Q_{0}}|g(y)|^{q} w(y) d y=q \int_{0}^{\infty} t^{q-1} w\left(\left\{x \in Q_{0}:|g(x)|>t\right\}\right) d t
$$

for all $g \in L_{w}^{q}\left(Q_{0}\right)$. We omit the details.

Now, Lemma 5.25 follows from Lemma 7.2 exactly as Corollary 5.22 from Lemma 5.21 .

Proof of Lemma 5.25. Since $\langle f\rangle_{Q_{0}}=0$, we have $f_{Q_{0}}|f| d y \leq M_{\mathrm{res}, Q_{0}, 1}^{\sharp} f(x)$ for all $x \in Q_{0}$. So

$$
w\left(Q_{0}\right)\left(f_{Q_{0}}|f| d x\right)^{q} \leq \int_{Q_{0}}\left|M_{\mathrm{res}, Q_{0}, 1}^{\sharp} f(x)\right|^{q} w(x) d x
$$

and the claim follows from Lemma 7.2 exactly as Corollary 5.22 from Lemma 5.21.

Acknowledgment. The authors thank Pekka Alestalo from the Helsinki University of Technology for bringing to their attention the notion of unbounded John domains.

\section{References}

[1] Acosta, G., R. G. DurÁn, and M. A. Muschietti: Solutions of the divergence operator on John domains. - Adv. Math. 206:2, 2006, 373-401.

[2] Adams, R. A.: Sobolev spaces. - Pure Appl. Math. 65, Academic Press, New York-London, 1975.

[3] Boman, J.: $L^{p}$-estimates for very strongly elliptic systems. - Technical Report 29, Department of Mathematics, University of Stockholm, Sweden, 1982.

[4] Buckley, S., P. Koskela, and G. Lu: Boman equals John. - In: XVIth Rolf Nevanlinna Colloquium (Joensuu, 1995), de Gruyter, Berlin, 1996, 91-99.

[5] ChuA, S.-K.: Weighted Sobolev inequalities on domains satisfying the chain condition. - Proc. Amer. Math. Soc. 117:2, 1993, 449-457.

[6] Coifman, R. R., and Y. Meyer: Au delà des opérateurs pseudo-différentiels. - Société Mathématique de France, Paris, 1978 (with an English summary).

[7] Cruz-Uribe, D., J. M. Martell, and C. PÉrez: Weights, extrapolation, and the theory of Rubio de Francia. - Work in progress (book).

[8] Cruz-Uribe, D., J. M. Martell, and C. PÉrez: Extensions of Rubio de Francia's extrapolation theorem. - In: Proceedings of the 7th International Conference on Harmonic Analysis and Partial Differential Equations (El Escorial, June 21-25, 2004), Collect. Math. Extra Vol., 2006, 195-231.

[9] De Guzmán, M.: Differentiation of integrals in $\mathbf{R}^{n}$. With appendices by Antonio Córdoba, and Robert Fefferman, and two by Roberto Moriyón, - Lecture Notes in Math. 481, Springer-Verlag, Berlin, 1975.

[10] Diening, L., and M. RŮŽIČKA: Calderón-Zygmund operators on generalized Lebesgue spaces $L^{p(\cdot)}$ and problems related to fluid dynamics. - J. Reine Angew. Math. 563, 2003, 197-220. 
[11] Diening, L., M. RƯŽIČKA, and J. Wolf: Existence of weak solutions for unsteady motions of generalized newtonian fluids. - Ann. Sc. Norm. Super. Pisa Cl. Sci. (5) (to appear).

[12] Fabes, E. B., C. E. Kenig, and R. P. Serapioni: The local regularity of solutions of degenerate elliptic equations. - Comm. Partial Differential Equations 7:1, 1982, 77-116.

[13] Fefferman, C., and E. M. Stein: $H^{p}$ spaces of several variables. - Acta Math. 129:3-4, 1972, $137-193$.

[14] García-Cuerva, J., and J. L. Rubio de Francia: Weighted norm inequalities and related topics. - North Holland, Amsterdam, 1985.

[15] Huber, A.: Die Divergenzgleichung in gewichteten Räumen und Flüssigkeiten mit $p(\cdot)-$ Struktur. - Master's thesis, University of Freiburg, Germany, 2005.

[16] Hurri, R.: Poincaré domains in $\mathbf{R}^{n}$. - Ann. Acad. Sci. Fenn. Ser. A I Math. Diss. 71, 1988, $1-42$.

[17] Hurri-SyrJänen, R.: A generalization of an inequality of Bhattacharya and Leonetti. Canad. Math. Bull. 39:4, 1996, 438-447.

[18] Hurri-Syrjänen, R.: A weighted Poincaré inequality with a doubling weight. - Proc. Amer. Math. Soc. 126:2, 1998, 545-552.

[19] Iwaniec, T.: On $L^{p}$-integrability in PDE's and quasiregular mappings for large exponents. Ann. Acad. Sci. Fenn. Ser. A I Math. 7, 1982, 301-322.

[20] Iwaniec, T., and C. A. Nolder: Hardy-Littlewood inequality for quasiregular mappings in certain domains in $\mathbf{R}^{n}$. - Ann. Acad. Sci. Fenn. Ser. A I Math. 10, 1985, 267-282.

[21] John, F.: Rotation and strain. - Comm. Pure Appl. Math. 14, 1961, 391-413.

[22] Krasnosel'skiǏ, M. A., and Ya. B. RutickiǏ: Convex functions and Orlicz spaces. - P. Noordhoff Ltd., Groningen, The Netherlands, 1961.

[23] Martio, O., and J. Sarvas: Injectivity theorems in plane and space. - Ann. Acad. Sci. Fenn. Ser. A I Mth. 4, 1978-79, 383-401.

[24] NÄKKI, R., and J. VÄISÄLÄ: John disks. - Expo. Math. 9:1, 1991, 3-43.

[25] Pileckas, K.: Recent advances in the theory of Stokes and Navier-Stokes equations in domains with non-compact boundaries. - In: Mathematical theory in fluid mechanics (Paseky, 1995), Pitman Res. Notes Math. Ser. 354, Longman, Harlow, 1996, 30-85.

[26] Rubio de Francia, J. L.: Factorization theory and $A_{p}$ weights. - Amer. J. Math. 106:3, 1984, $533-547$.

[27] Schumacher, K.: Solutions to the equation $\operatorname{div} u=f$ in weighted Sobolev spaces. - Technical Report 2511, FB Mathematik, TU Darmstadt, 2007.

[28] Shvartsman, P.: On extensions of Sobolev functions defined on regular subsets of metric measure spaces. - J. Approx. Theory 144:2, 2007, 139-161.

[29] Simader, C. G., and H. Sohr: The Dirichlet problem for the Laplacian in bounded and unbounded domains. - Pitman Res. Notes Math. Ser. 360, Longman, Harlow, 1996.

[30] Stein, E. M.: Singular integrals and differentiability properties of functions. - Princeton Univ. Press, Princeton, N.J., 1970.

[31] Stein, E. M.: Harmonic analysis: real-variable methods, orthogonality, and oscillatory integrals. - Princeton Univ. Press, Princeton, NJ, 1993. 